\title{
A goal programming approach for fuzzy flexible linear programming problems
}

\author{
S.H. Nasseri ${ }^{1, *}$ and G.A. Ramzannia-Keshteli ${ }^{2}$
}

\begin{abstract}
We are concerned with solving Fuzzy Flexible Linear Programming (FFLP) problems. Even though, this model is very practical and is useful for many applications, but there are only a few methods for its situation. In most approaches proposed in the literature, the solution process needs at least, two phases where each phase needs to solve a linear programming problem. Here, we propose a method to solve the given problem in just one phase using only one problem. Furthermore, using our approach, sensitivity analysis of Fuzzy Flexible Linear Programming (FFLP) problem is simpler. For an illustration of our method, some numerical examples given. In particular, a practical problem is formulated and is solved by our method and several other methods and the obtained results are compared.
\end{abstract}

Keywords: Linear programming, goal programming, fuzzy flexible linear programming, multi parametric linear programming, sensitivity analysis.

Manuscript was received on 10/11/2017, revised on 02/04/2018 and accepted for publication on 04/09/2018.

\section{Introduction}

Linear Programming (LP) problems has been used to model many serious real-life decision making problems such as management, economic, transportation, data envelopment analysis, railways, agricultural, and many industrial applications [24,25]. LP problems involve the optimization of a linear objective function, subject to some linear equality and inequality constraints involving some continuous /discrete non-negative decision variables. A typical model of an LP problem is:

$$
\begin{aligned}
& \operatorname{Min} \quad Z(x)=\sum_{j=1}^{n} c_{j} x_{j} \\
& \text { s.t. } \quad \sum_{j=1}^{n} a_{i j} x_{j} \geq b_{i}, \quad i=1,2, \ldots, m \\
& x_{j} \geq 0, \quad j=1,2, \ldots, n \text {. }
\end{aligned}
$$

and Goal Programming (GP) as a multi-objective optimization approach in turn as multi-criteria decision analysis was introduced originally by Charnes and Cooper [8] to solve multi-objective programming problems with multiple and conflicting objectives. Later, Lee [19], Ignizio [18], Romero [27], Chang [6-7], Tabrizi et al. [31], Narasimhan [22] used GP in various areas such as engineering, transportation, economics, agriculture, and finance. GP is an important technique for Decision-Makers (DMs) considering simultaneously several objectives in finding a set of desirable solutions.

\footnotetext{
* Corresponding Author.

${ }^{1}$ Department of Mathematics, University of Mazandaran, Babolsar, Iran, Email: nhadi57@gmail.com.

${ }^{2}$ Department of Mathematics, University of Mazandaran, Babolsar, Iran, Email: gh.ramezan@stu.umz.ac.ir
} 
Decision makers have applied fuzzy set theory to solve some complex problems in management science, and industrial applications. The idea of fuzzy mathematical programming was first proposed by Tanaka et al. [32] in the framework of fuzzy decision of Bellman and Zadeh [3]. For the first time, Zimmerman [39] presented a formulation of fuzzy LP problem and subsequently different types of LP problems were proposed by many authors to solve complex real-life problems. Recently, Bector and Chandra [2] presented four categories of FLP problems to be mention in the following section:

Type 1: LP problems with fuzzy inequalities and crisp objective function Type 2: LP problems with crisp inequalities and fuzzy objective function Type3: LP problems with fuzzy inequalities and fuzzy objective function Type4: LP problems with fuzzy parameters

In the type 1 FLP problems is presumed that the fuzziness of the available resources is characterized by the membership function over a tolerance range.

It is necessary to differentiate between flexibilities in constraints and the goal and uncertainty of the data. Flexibility is modeled by fuzzy sets and may reflect the fact that constraints or goal are linguistically formulated, and their satisfaction is a matter of tolerance and degrees or fuzziness [3]. On the other hand, there is ambiguity corresponding to an objective variability in the model parameters (Randomness), or a lack of knowledge of the parameter values (epistemic uncertainty). Randomness originates from the random nature of events and it is about uncertainty regarding the membership or non-membership of an element in a set. Epistemic uncertainty deals with ill-known parameters modeled by fuzzy intervals in the setting of possibility theory [13, 38]. In [33], Verdegay proposed a parametric linear programming model with a single parameter using $\alpha$-cuts to achieve an equivalent model for fuzzy linear programming problems with flexible constraints. After that, Verdegay [34] used the duality results to solve the original fuzzy linear programming problem. Werner's in [36] introduced an interactive multiple objective programming model subject to its constraint being flexible and proposed a special approach for solving multiple objective programming models based on fuzzy set theory. In the mentioned work, the classical model is extended by integrated the flexible constraints. After that, Delgado et al. [11] proposed a general model for fuzzy linear programming problem. In particular, the authors suggested a resolution method for the mentioned problem. Campos et al. [9] considered a linear programming problem with fuzzy constraints including fuzzy coefficients in both the matrix and the right hand sides. They dealt with an auxiliary model resulting from the embedding constraints of the main model. After that, Nasseri et al [23] introduced an equivalent fuzzy linear model for the flexible linear programming problems and proposed a fuzzy primal simplex algorithm to solve these problems. Recently, Attari and Nasseri [1] introduced a concept of feasibility and efficiency of solution for the fuzzy mathematical programming problems. The suggested algorithm solves two classical associated linear programing problems to achieve an optimal flexible solution. Unfortunately, this process is not efficient and the sensitivity analysis on this model, does not work well, because the initial fuzzy flexible linear programming problem after being transfered to a multi-parametric linear programming problem is reduced to two associated linear programming problems. Here, we are going to overcome the mentioned shortcomings by first introducing an associated multi-parametric linear programming problem and then proposing a new goal programming approach to obtain an optimal solution efficiently.

The rest of our work is organized as follows. In Section 2, a practical form of fuzzy linear programming model, entitled fuzzy flexible linear programming problem, is introduced. In 
particular, a two-phase algorithm is presented to solve these models. In Section 3, first based on the fuzzy flexible linear programming model, a multi-parametric linear programming problem is introduced and then a new approach for solving the associated problem is given. We entitled the model as MPLGP problem. Section 4 gives a comparative study of the mentioned methods given in sections 2 and 3. A post optimality discussion based on MPLGP model is given in Section 5 to complete our discussion. Finally, Section 6 considers a case study concerned with producing four products of a manufacturing company to cultivate some strategic products to achieve the maximum profit.

\section{Fuzzy flexible linear programming}

A convenient fuzzy linear programming model is a Fuzzy Flexible Linear Programming (FFLP) problem. There are various the flexible linear programming $[1,10,23]$ models in the literature. In the following subsection, a definition of problem is given.

\subsection{FFLP problem with linear membership function}

Consider a decision maker faced with a linear programming problem in which he/she can endure violation in satisfaction of at the constraints. For each constraint in the constraint set, this assumption can be denoted by $a_{i} x \preccurlyeq^{F} b_{i}, i=1,2, \ldots, n$, modeled by use of a membership function as follows:

$$
\mu_{i}(x)=\left\{\begin{array}{lr}
1, & a_{i} x \leq b_{i} \\
f_{i}\left(a_{i} x\right), & b_{i} \leq a_{i} x \leq b_{i}+p_{i} \\
0, & a_{i} x \geq b_{i}+p_{i}
\end{array}\right.
$$

where $f_{i}($.$) is strictly decreasing and continuous, f_{i}\left(b_{i}\right)=1$ and $f_{i}\left(b_{i}+p_{\boldsymbol{i}}\right)=0$.

This membership function expresses that the decision maker tolerates violation in the accomplishment of the constrain $i$ up to the value $b_{i}+p_{i}$. The function $\mu_{i}(x)$ gives the degree of satisfaction of the $i$ th constrains for $x \in \mathbb{R}^{n}$, but this value is obtained by means of the function $f_{i}$, defined over $\mathbb{R}$. Considering these assumptions, the associated FFLP problem can be presented as follows:

$$
\begin{aligned}
& \operatorname{Max} \tilde{Z}(x)=\tilde{C} x \quad \operatorname{Max} \quad \tilde{Z}(x)=\sum_{j=1}^{n} \tilde{c}_{j} x_{j} \\
& \text { s.t. } \quad A x \preccurlyeq^{F} b \Leftrightarrow \text { s.t. } \sum_{j=1}^{n} a_{i j} x_{j} \preccurlyeq^{F} b_{i}, i=1,2, \ldots, m \text {, } \\
& x \geq 0 \quad x_{j} \geq 0, j=1,2, \ldots, n, \\
& \operatorname{Max} \tilde{Z}(x)=\sum_{j=1}^{n} \tilde{c}_{j} x_{j} \\
& \Leftrightarrow \text { s.t. } H_{i}\left(x, a_{i}\right)=\sum_{j=1}^{n} a_{i j} x_{j}-b_{i} \preccurlyeq^{F} 0, i=1,2, \ldots, m \text {, } \\
& x_{j} \geq 0, \quad j=1,2, \ldots, n \text {, }
\end{aligned}
$$

where $x=\left(x_{1}, \ldots, x_{n}\right)^{T}$ is an $n$-dimensional real decision vector, $\tilde{C}=\left(\tilde{c}_{1}, \tilde{c}_{2}, \ldots, \tilde{c}_{n}\right)$ is an $n$-dimensional fuzzy vector of fuzzy parameters involved in the objective function $Z$. Here, the fuzzy parameters are assumed to be characterized by fuzzy numbers as introduced in [33]. 
For these fuzzy parameters, the membership function $\mu_{\tilde{c}_{j}}, j=1,2, \ldots, n$, are defined for a fuzzy number $\tilde{c}_{j}$, where $\tilde{c}_{j}$ is a convex continuous fuzzy subset on the real line. $a_{i}$ is the $i$ th row of matrix $A=\left[a_{i j}\right]_{m \times n^{\prime}}, j=1,2, \ldots, n, i=1,2, \ldots, m$, a real $m \times n$ dimensional matrix of technical coefficients. The notation "max", is used for maximizing the objective function in a fuzzy sense, and " $\lessgtr$ " represents a fuzzy extension of " $\leq$ " on $\mathbb{R}$ which is applied to compare the left side of the fuzzy constraints with the right hand side [33] .

In general, model (3) is not well-defined due to the following reasons:

i. We cannot maximize the fuzzy quantity $\tilde{z}(x)$.

ii. The constraint $H_{i}\left(x, a_{i}\right) \preccurlyeq^{F} b_{i}, i=1,2, \ldots, m$, do not result in a crisp feasible set.

Now, we are going to discuss on fuzzy ordering based on using linear ranking functions. One appropriate approach to state a crisp optimal solution preference of alternative is comparing fuzzy quantities by means of ranking function $\mathfrak{R}: F(\mathbb{R}) \rightarrow \mathbb{R}$ that maps each fuzzy quantity to the real line, where there exists a natural order (for more details, see in $[20,25]$ ).

If, we want to define a deterministic feasible set, an idea is to provide confidence level $\alpha_{i}$ at which it is desired that the corresponding $i$ th fuzzy constraint holds. Therefore, in order to remove those mentioned restriction, the following problem is devised:

$$
\begin{array}{ll}
\text { Max } & Z(x)=\Re(z(\tilde{c}, x)) \\
\text { s.t. } & \mu_{i}\left\{H_{i}\left(x, a_{i}\right) \preccurlyeq F^{F} 0\right\} \geq \alpha_{i} \\
& \alpha_{i} \geq \alpha_{i}^{D}, 0 \leq \alpha_{i} \leq 1, i=1,2, \ldots, m \\
& x \geq 0,
\end{array}
$$

Where $\mathfrak{R}(z(\tilde{c}, x))$ means the corresponding crisp value of the fuzzy function $z(\tilde{c}, x)$ based on a linear ranking function. To drive for a meaningful choice of membership function for each fuzzy constraint, it is disputed that if $H_{i}\left(x, a_{i}\right) \leq 0$, then the $i$ th constraint is fully satisfied. If $H_{i}\left(x, a_{i}\right) \geq$ $p_{i}$, where $p_{i}$ is the predefined maximum tolerance from zero, as determined by the decision maker, then the $i$ th constraint is perfectly violated. For $H_{i}\left(x, a_{i}\right) \in\left(0, p_{i}\right)$, the membership function is monotonically decreasing. If this decrease is along with a linear function, then it is sensible to select the membership function of the $i$ th constraint as

$$
\mu_{i}\left\{H_{i}\left(x, a_{i}\right) \preccurlyeq 0\right\}=\left\{\begin{array}{cc}
1, & H_{i}\left(x, a_{i}\right) \leq 0 \\
1-\frac{H_{i}\left(x, a_{i}\right)}{p_{\boldsymbol{i}}}, & 0 \leq H_{i}\left(x, a_{i}\right) \leq p_{\boldsymbol{i}} \\
0, & H_{i}\left(x, a_{i}\right) \geq 0 .
\end{array}\right.
$$

This relation can be rewrites as follows:

and (4) gets to be

$$
\mu_{i}(x)=\left\{\begin{array}{cc}
1, & a_{i} x \leq b_{i} \\
\frac{b_{i}+p_{i}-a_{i} x}{p_{i}}, & b_{i} \leq a_{i} x \leq b_{i}+p_{i} \\
0, & a_{i} x \geq b_{i}+p_{i}
\end{array}\right.
$$

$$
\begin{array}{ll}
\text { Max } & Z(x)=\Re(z(\tilde{c}, x)) \\
\text { s.t. } & a_{i} x \leq b_{i}+p_{i}\left(1-\alpha_{i}\right)
\end{array}
$$




$$
\begin{gathered}
\alpha_{i} \geq \alpha_{i}^{D}, 0 \leq \alpha_{i} \leq 1, i=1,2, \ldots, m, \\
x \geq 0 .
\end{gathered}
$$

We name the above problem as multi-parametric linear programming problem and we will show it in an abbreviated form as (MPLP1) $[1,4,16]$.

Now, we are going to give the concept of feasible solution of the fuzzy linear programming problem (7).

Definition 1. Let $\bar{\alpha}=\left(\alpha_{1}, \ldots, \alpha_{m}\right) \in(0,1]^{m}$ be a vector, and

$$
X_{\bar{\alpha}}=\left\{x \in \mathbb{R}^{n} \mid x \geq 0, a_{i} x \leq b_{i}+p_{i}\left(1-\alpha_{i}\right), \alpha_{i} \geq \alpha_{i}^{D}, i=1,2, \ldots, m\right\} .
$$

A vector $x \in X_{\bar{\alpha}}$ is called an $\bar{\alpha}$-feasible solution to problem (7).

The following proposition allows us to define the feasible set for problem (7) as an intersection of all $\bar{\alpha}$-cuts corresponding to fuzzy constraints.

Proposition 1. Let $\bar{\alpha}=\left(\alpha_{1}, \ldots, \alpha_{m}\right) \in(0,1]^{m}$. Then $X_{\bar{\alpha}}=\bigcap_{i=1}^{m} X_{\alpha_{i}}^{i}$, where

$$
X_{\alpha_{i}}^{i}=\left\{x \in \mathbb{R}^{n} \mid x \geq 0, \alpha_{i} \geq \alpha_{i}^{D}, a_{i} x \leq b_{i}+p_{i}\left(1-\alpha_{i}\right)\right\}
$$

For $i=1,2, \ldots, m$ (namely, $X_{\alpha_{i}}^{i}$ is the $\alpha$-cut of the $i$ th fuzzy constraint).

Proof. For any $\bar{\alpha}=\left(\alpha_{1}, \ldots, \alpha_{m}\right) \in(0,1]^{m}$, let $x \in X_{\bar{\alpha}}$. Then, $\alpha_{i} \geq \alpha_{i}^{D}, a_{i} x \leq b_{i}+p_{i}\left(1-\alpha_{i}\right)$. Now from (9), we have $x \in X_{\alpha_{i}}^{i}, i=1,2, \ldots, m$, and therefore $x \in \bigcap_{i=1}^{m} X_{\alpha_{i}}^{i}$. On the other hand, if $x \in \bigcap_{i=1}^{m} X_{\alpha_{i}}^{i}$, then we have $x \in X_{\alpha_{i}}^{i}$, for all $i=1,2, \ldots, m$. Therefore, $\alpha_{i} \geq \alpha_{i}^{D}, a_{i} x \leq b_{i}+$ $p_{i}\left(1-\alpha_{i}\right) \quad$ and hence $\quad x \in X_{\bar{\alpha}}$. This completes the proof.

Proposition 2. Let $\overline{\alpha^{\prime}}=\left(\alpha^{\prime}{ }_{1}, \ldots, \alpha^{\prime}{ }_{m}\right)$ and $\bar{\alpha}^{\prime \prime}=\left(\alpha^{\prime \prime}{ }_{1}, \ldots, \alpha^{\prime \prime}{ }_{m}\right)$, where $\alpha_{i}^{\prime} \leq \alpha_{i}^{\prime \prime}$ for all. Then $\bar{\alpha}^{\prime \prime}$-feasibility of $x$ implies $\overline{\alpha^{\prime}}$-feasibility.

Proof. The proof is straightforward.

For a given $\alpha \in(0,1]$, let $x \in \mathbb{R}^{n}$ be a usual $\alpha$-feasible solution to (7) (a solution with the same degrees of satisfaction in all of constraint). It has the meaning of $a_{i} x \leq b_{i}+p_{i}\left(1-\alpha_{i}\right), \alpha_{i} \geq \alpha_{i}^{D}$ orequivalently $x \in X_{\alpha}^{i}$,for all $i=1,2, \ldots, m$.

If $\bar{\alpha}=(\alpha, \ldots, \alpha) \in(0,1]^{m}$, then $x \in X_{\alpha}$ which impliesthat the $\alpha$-feasibility of (7) can be understood as a special case of the $\bar{\alpha}$-feasibility.So the following result can be obtained.

Corollary 1. If problem (7) is not infeasible, then $X_{\alpha}$ is not empty.

Proof. The proof is straightforward.

Definition 2. Let" $<F$ " be a fuzzy extension of the binary relation " $\leq$ " and let $x^{*}=\left(x_{1}{ }^{*}, \ldots, x_{n}{ }^{*}\right)^{T} \in$ $\mathbb{R}^{n}$ be an $\bar{\alpha}$-feasibility solution to (7), where $\bar{\alpha}=\left(\alpha_{1}, \ldots, \alpha_{m}\right) \in(0,1]^{m}$ and let $Z(\tilde{c}, x)$ be a fuzzy objective. The vector $x \in \mathbb{R}^{n}$ is an $\bar{\alpha}$-efficient solution to (7) with maximization of the objective function, if there is no any $x^{\prime} \in X_{\bar{\alpha}}$ such that $\mathfrak{R}(Z(\tilde{c}, x))<\mathfrak{R}\left(Z\left(\tilde{c}, x^{\prime}\right)\right)$.

An $\bar{\alpha}$-efficient solution with minimization of the objective function can be defined similarly. 
Note that any $\bar{\alpha}$-efficient solution to the FFLP problem is an $\bar{\alpha}$-feasible solution to the FFLP problem with some extra properties. In the following theorem, we present a necessary and sufficient condition for an $\bar{\alpha}$-efficient solution to (7).

Theorem 1. Let $\bar{\alpha}=\left(\alpha_{1}, \ldots, \alpha_{m}\right) \in(0,1]^{m}$ and $x^{*}=\left(x_{1}{ }^{*}, \ldots, x_{n}{ }^{*}\right)^{T}, x_{j}^{*} \geq 0, j=1,2, \ldots, n$ be an $\bar{\alpha}$-feasible solution to (7). Then a vector $x^{*} \in \mathbb{R}^{n}$ is an $\bar{\alpha}$-efficient solution to problem (7) with maximization of the objective function, if and only if $x^{*}$ is an optimal solution of the following problem:

$$
\begin{gathered}
\operatorname{Max} \quad Z(x)=\mathfrak{R}(z(\tilde{c}, x)) \\
\text { s.t. } \quad a_{i} x \leq b_{i}+p_{i}\left(1-\alpha_{i}\right), i=1,2, \ldots, m, \\
\alpha_{i} \geq \alpha_{i}^{D}, 0 \leq \alpha_{i} \leq 1, i=1,2, \ldots, m, \\
x_{j} \geq 0, j=1,2, \ldots, n,
\end{gathered}
$$

where each $p_{i}$ is a predefined maximum tolerance.

Proof. Let $\bar{\alpha}=\left(\alpha_{1}, \ldots, \alpha_{m}\right) \in[0,1]^{m}$ and $x^{*}=\left(x_{1}{ }^{*}, \ldots, x_{n}{ }^{*}\right)^{T}, x_{j}^{*} \geq 0, j=1,2, \ldots, n$ be an $\bar{\alpha}$-efficient solution to problem (7) with maximization of the objective function. By Definition 1 and (6), we have $a_{i} x^{*} \leq b_{i}+p_{i}\left(1-\alpha_{i}\right), \alpha_{i} \geq \alpha_{i}^{D}$ for $i=1,2, \ldots, m$. Therefore, $x^{*}$ is a feasible solution to (10). Also by Definition 8 , there is no $x^{\prime} \in X_{\bar{\alpha}}$ such that $\mathfrak{R}\left(Z\left(\tilde{c}, x^{*}\right)\right)<\Re\left(Z\left(\tilde{c}, x^{\prime}\right)\right)$. Thus means that $x^{*}$ is an optimal solution to (10), and in this case $x^{*}$ is obviously an $\bar{\alpha}$-feasible solution to the problem (7). Thus by Definition 2, the optimality of $x^{*}$ implies the $\bar{\alpha}$-efficiency of $x^{*}$.

In Theorem1, we have provided a computational method to solve fuzzy flexible linear programming problem (7). Thus by assigning a specific $\bar{\alpha}$ by a decision maker, we may replace the $\alpha_{j}$ in the corresponding constraint of (10), and solve the resulted problem to compute the $\bar{\alpha}$-efficient solution to problem (7). An $\bar{\alpha}$-efficient solution to (7) has two characteristics:

i. The solution has various satisfaction degrees corresponding to the constraints.

ii. The acquired solution is optimal.

This solution permits the decision maker to obtain a more flexible and more compatibility by assigning desired preferences, specially in an online optimization process.

In Theorem 1, a method to FFLP problem is introduced to obtain an $\bar{\alpha}$-efficient solution. If the resulting problem (10) has only one optimal solution, then we know this solution is an $\bar{\alpha}$-efficient solution to the given fuzzy problem. In the case of that problem (10) has some multiple optimal solutions, find a maximum efficient solution, i.e., an $\bar{\alpha}^{\prime}$-efficient solution with $\alpha^{\prime} \geq \alpha, i=$ $1,2, \ldots, m$, we apply the following two-phase approach.

In the two-phase approach, problem (10) is solved in phase 1, while in phase 2, a solution is obtained which has a higher satisfaction degree than the previous solution. So, by using the twophase approach, we can obtain a better utilization of the available resources. Also, the solution resulting by this two-phase approach is always an $\bar{\alpha}^{\prime}$-efficient solution.

We will call problem (10) as the phase 1 problem. Let $\bar{\alpha}^{0}=\left(\alpha^{0}{ }_{1}, \ldots, \alpha^{0}{ }_{m}\right)$ and $\left(x^{*}, \Re\left(Z\left(\tilde{c}, x^{*}\right)\right)\right)$ be the optimal solution of Phase 1 with $\bar{\alpha}^{0}$ degree of efficiency. Set $\alpha_{i}^{*}=\mu_{i}\left\{H_{i}\left(x^{*}, a_{i}\right) \prec^{F} 0\right\} \geq$ $\alpha_{i}^{0}$. In Phase 2, we solve the following problem: 


$$
\begin{gathered}
\operatorname{Max} W_{\alpha}=\sum_{i=1}^{m} \alpha_{i} \\
\text { s.t. } \quad \mathfrak{R}(Z(\tilde{c}, x)) \geq \Re\left(Z\left(\tilde{c}, x^{*}\right)\right) \\
a_{i} x \leq b_{i}+p_{i}\left(1-\alpha_{i}\right), \\
\alpha_{i}^{*} \leq \alpha_{i} \leq 1, i=1,2, \ldots, m, \\
x \geq 0,
\end{gathered}
$$

We name problem (11) as Multi-Parametric Linear Programming problem (MPLP2).

Let $\left(x^{* *}, \alpha_{1}^{* *}, \ldots, \alpha_{m}^{* *}\right)$ be an optimal solution to (MPLP 2) as given by (11). We have the following result.

Theorem 2. $x^{* *}$, an optimal solution of problem (11) (MPLP 2), is a maximal $\bar{\alpha}$-efficient solution of problem (7).

Proof. Since in problem (11), we have $\alpha_{i}{ }^{*} \geq \alpha_{i}^{0}$, using Proposition 2, $x^{* *}$ is an $\bar{\alpha}^{0}$-feasible solution to problem (3) and this implies that it is feasible for problem (10). Now, by optimality of $x^{*}$ in (11) and $\mathfrak{R}\left(Z\left(\tilde{c}, x^{* *}\right)\right) \geq \mathfrak{R}\left(Z\left(\tilde{c}, x^{*}\right)\right)$, we have optimality of $x^{* *}$ in (10) and hence $\mathfrak{R}\left(Z\left(\tilde{c}, x^{* *}\right)\right)=\mathfrak{R}\left(Z\left(\tilde{c}, x^{*}\right)\right)$. Therefore, $x^{* *}$ is also an $\bar{\alpha}^{0}$-efficient solution to (7). Also because $\left(x^{* *}, \alpha_{1}^{* *}, \ldots, \alpha_{m}^{* *}\right)$ is optimal and the coefficients in the objective function of problem (11) are positive, we have $\alpha_{i}^{* *}=\mu_{i}\left\{H_{i}\left(x^{*}, a_{i}\right) \preccurlyeq^{F} 0\right\}, i=1,2, \ldots, m$. Now, assume $x^{* *}$ be not a maximum $\bar{\alpha}^{0}$-efficient solution to (7). Then, there exists an $\bar{\alpha}^{0}$-efficient $x^{\prime}$ to (7) such that $\alpha_{i}{ }^{\prime} \geq \alpha_{i}^{* *}, i=$ $1,2, \ldots, m$ and for some $k$, we have $\alpha_{K}{ }^{\prime} \geq \alpha_{K}^{* *}$, where $\alpha_{i}{ }^{\prime}=\mu_{i}\left\{H_{i}\left(x, a_{i}\right) \preccurlyeq^{F} 0\right\}, i=1,2, \ldots, m$ and $\mathfrak{R}\left(Z\left(\tilde{c}, x^{\prime}\right)\right) \geq \mathfrak{R}\left(Z\left(\tilde{c}, x^{*}\right)\right)$. Therefore, we have $\left(x^{\prime}, \alpha_{1}^{\prime}, \ldots, \alpha_{m}^{\prime}\right)$ as a feasible solution of (11) and

$$
\sum_{i=1}^{m} \alpha_{i}^{* *}=\sum_{i=1, i \neq k}^{m} \alpha_{i}^{* *}+\alpha_{k}^{* *}<\sum_{i=1, i \neq k}^{m} \alpha_{i}^{\prime}+\alpha_{k}^{\prime}=\sum_{i=1}^{m} \alpha_{i}^{\prime} .
$$

But this implies that $\left(x^{* *}, \alpha_{1}^{* *}, \ldots, \alpha_{m}^{* *}\right)$ is not a solution to (11) and it is clearly a contradiction.

Now, we are ready to present our algorithm for solving FFLP problem.

\subsection{Algorithm 1. Main steps of the proposed algorithm for FFLP problem}

Assumption 1: A fuzzy mathematical model in the form of Fuzzy Flexible Linear Programming (FFLP) is given. The parameters of the equivalent model appeared in (7) including $a_{i}, b_{i}, p_{i}$ and $\tilde{c}_{j}$, for $j=1,2, \ldots, n$, and $\alpha_{i}^{D}$, for all $i=1,2, \ldots, m$ are given).

Assumption 2. A linear ranking function to rank the fuzzy numbers is given (see $[2,3,4,6,10,11$, $12,13,14,21,22,24,25$, and 26]).

Step 1: Using the given ranking function, obtain the corresponding crisp objective function for the given fuzzy objective function.

Step 2: Obtain the corresponding Multi-Parametric Linear Programming problem (MPLP1) for problem (3), based on the relations (4)-(6).

Step 3: Solve the MPLP 1 and first obtain the optimal value of $x^{*}$ and $\alpha^{*}$, and then the optimal value of the objective function $\left(z^{*}\right)$. 
Step 4: Reformat MPLP1 in the format of MPLP 2 based on the optimal solution of MPLP1 in step 3

Step 5: Solve MPLP2 to obtain an optimal solution for the FFLP, considered as the main problem (3).

\section{Goal programming approach for solving FFLP problem}

\subsection{A survey of goal programming model}

The goal programming originally proposed by Charnes and Cooper [8] is used to solve multiobjective decision making problems by finding solutions being satisfactory. GP is one of the most powerful multi-objective optimization techniques, based on the distance function, where a decisionmaker (DM) looks for the solution that minimizes the absolute deviation between the achievement level of the objective and its aspiration level. This can be stated as follows:

$$
\text { Min } D=\sum_{k=1}^{K}\left|G_{k}(x)-\mathrm{g}_{k}\right|
$$

s.t. $x \in$ FSS (FSS is the Feasible Solution Set)

where $G_{k}(x)$ is the linear function of the $k$ th goal, and $\mathrm{g}_{k}$ is the aspiration level of the $k$ th goal. In $\mathrm{GP}$, the distance between $G_{k}(x)$ and $\mathrm{g}_{k}, d_{k}\left(G_{k}(x), \mathrm{g}_{k}\right)=\left|G_{k}(x)-\mathrm{g}_{k}\right|$ is expressed by the deviational variable $d_{k}^{+}$and $d_{k}^{-}(k=1, \ldots, K)$, where $d_{k}^{-}$is the negative deviational variable, $d_{k}^{-}=\max \left(0, \mathrm{~g}_{k}-G_{k}(x)\right)$ and $d_{k}^{+}$is the positive deviational variable, $d_{k}^{+}=\max \left(0, G_{k}(x)-\mathrm{g}_{k}\right)$. Minimizing the distance between $G_{k}(x)$ and $d_{k}^{+}$means minimizing either $d_{k}^{-}$or $d_{k}^{+}$or $d_{k}^{-}+d_{k}^{+}$.

We minimize $d_{k}^{-}$, when we need $G_{k}(x) \geq \mathrm{g}_{k}$ (maximization problem), minimize $d_{k}^{+}$, when we need $G_{k}(x) \leq \mathrm{g}_{k}$ (minimization problem) and also minimize $d_{k}^{-}+d_{k}^{+}$, when we need $G_{k}(x) \leq \mathrm{g}_{k}$.

Accordingly, GP is a minimization problem of the deviational variables which can be done by various type of methods such as Standard GP (SGP), weighted GP (WGP), Lexicographic GP (LGP), Chebyshev or min-max GP (MGP), Mixed binary GP (MBGP), Fuzzy GP (FGP), MultiChoice as GP (MCGP) and Fuzzy Multi-Choice GP (FMCGP) are some extensions of the basic GP model (Lee 1978; Ignizio 1976; Zimmerman 1978; Tamiz, Jones and Romero 1998; Romero 2001; Chang 2004, 2007; Bankian Tabrizi, Kamran Shahnaghi and Saeed Jabalameli 2012). Next, the mathematical formulations of the above models are presented as 1-8 below:

\section{Standard goal programming (SGP) model}

$$
\begin{gathered}
\operatorname{Min} D=\sum_{k=1}^{K} d_{k}^{-}+d_{k}^{+} \\
\text {s.t. } \quad G_{k}(x)-d_{k}^{+}+d_{k}^{-}=\mathrm{g}_{k}, k=1, \ldots, K, \\
x \in F S S, d_{k}^{+} \geq 0, d_{k}^{-} \geq 0, k=1, \ldots, K .
\end{gathered}
$$

\section{Lexicographic goal programming (LGP) model}




$$
\begin{gathered}
\operatorname{Min} a=\left[\sum_{k \in K_{1}}\left(w_{k}^{+} d_{k}^{+}+w_{k}^{-} d_{k}^{+}\right), \ldots, \sum_{k \in K_{r}}\left(w_{k}^{+} d_{k}^{+}+w_{k}^{-} d_{k}^{+}\right), \ldots, \sum_{k \in K_{q}}\left(w_{k}^{+} d_{k}^{+}+w_{k}^{-} d_{k}^{+}\right)\right] \\
\text {s.t. } \quad G_{k}(x)-d_{k}^{+}+d_{k}^{-}=\mathrm{g}_{k}, k=1, \ldots, K, K_{1}+\ldots+K_{r}+\ldots+K_{q}=K \\
x \in F S S, \quad d_{k}^{+} \geq 0, d_{k}^{-} \geq 0,
\end{gathered}
$$

where $K$ represents the index set of goals placed in the $r$ th priority level, $w_{k}^{+}$and $w_{k}^{-}$are the respective positive and negative weights attached to these deviations in the achievement function.

\section{Weighted goal programming (WGP) model}

$$
\begin{gathered}
\text { Min } D=\sum_{k=1}^{K} w_{k}^{-} d_{k}^{-}+w_{k}^{+} d_{k}^{+} \\
\text {s.t. } \quad G_{k}(x)-d_{k}^{+}+d_{k}^{-}=\mathrm{g}_{k}, \quad k=1, \ldots, K, \\
\\
x \in F S S, \quad d_{k}^{+} \geq 0, d_{k}^{-} \geq 0, \quad k=1, \ldots, K,
\end{gathered}
$$

where $w_{k}^{+}$and $w_{k}^{-}(k=1, \ldots, K)$ are determined by the decision maker.

\section{Chebyshev or min-max goal programming (MGP) model}

$$
\begin{gathered}
\text { Min } D=a \\
\text { s.t. } a \geq w_{k}^{-} d_{k}^{-}+w_{k}^{+} d_{k}^{+}, \\
G_{k}(x)-d_{k}^{+}+d_{k}^{-}=\mathrm{g}_{k}, \quad k=1, \ldots, K, \\
x \in F S S, \quad d_{k}^{+} \geq 0, d_{k}^{-} \geq 0, \quad k=1, \ldots, K,
\end{gathered}
$$

where $a$ is an extra continuous variable that measures the maximal deviation.

\section{Mixed binary goal programming (MBGP) model}

$$
\begin{gathered}
\operatorname{Min} D=\sum_{k=1}^{K}\left(d_{k}^{-}+d_{k}^{+}\right) \mathrm{b}_{k} \\
\text { s.t. } \quad G_{k}(x)-d_{k}^{+}+d_{k}^{-}=\mathrm{g}_{k}, \quad k=1, \ldots, K, \\
x \in F S S, \quad d_{k}^{+} \geq 0, d_{k}^{-} \geq 0, \quad k=1, \ldots, K,
\end{gathered}
$$

where the binary control variable of the $k$ th goal is $\mathrm{b}_{k}$, and $\mathrm{R}_{k}$ is the environment constraint function of the $k$ th goal.

\section{Fuzzy goal programming (FGP) model}

$$
\begin{gathered}
G_{k}(x) \lesssim \mathrm{g}_{k}\left(G_{k}(x) \gtrsim \mathrm{g}_{k}\right), k=1, \ldots, K . \\
\text { s.t. } \\
\text {. }
\end{gathered}
$$


where $G_{k}(x) \lesssim(\succsim) \mathrm{g}_{k}$ indicates the $k$ th fuzzy goal approximately less than or equal to approximately greater than or equal to) the aspiration level $\mathrm{g}_{k}$.

\section{Multi-choice goal programming (MCGP) model}

$$
\operatorname{Min} \sum_{k=1}^{K} \mid G_{k}(x)-\left(\mathrm{g}_{k 1} \text { or } \mathrm{g}_{k 2} \text { or ...or } \mathrm{g}_{k m}\right) \mid
$$

where all the variables are defined as in GP.

\section{Fuzzy multi-choice goal programming (FMCGP) model}

$$
\begin{array}{ll}
\text { Min } & \sum_{k=1}^{K} W_{k} \mid G_{k}(x)-\left(\tilde{\mathrm{g}}_{k 1} \text { or } \tilde{\mathrm{g}}_{k 2} \text { or } \ldots \text { or } \tilde{\mathrm{g}}_{k m}\right) \mid \\
\text { s.t. } & x \in F S S
\end{array}
$$

where $w_{k}, k=1, \ldots, K$ are the relative importance of the objective function and the aspiration level $\tilde{\mathrm{g}}_{k l}, l=1, \ldots, m$, are assumed to be triangular fuzzy numbers with membership functions $\mu_{k l}, l=$ $1, \ldots, m$.

\subsection{Proposed method (GP approach) for solving FFLP}

According to the given discussion in section 2, we observe that the proposed approach to solve FFLP problems is just to consider the intended amount of $\alpha_{i}$, and then obtain the amount of the objective function, and identify the maximum amount of objective function with the maximum value for the $\alpha_{i}$ through obtained feasible solutions. This solution is considered as an important initial information for the second phase, by keeping the optimum level of the current objective function and increasing the amount $\sum_{i=1}^{m} \alpha_{i}$.

In fact, two goals to be accomplished:

First goal: keep the value of obtained objective function and improve it.

Second goal: increase the value of $\sum_{i=1}^{m} \alpha_{i}$.

However, the above approach has following difficulties

1. If the value of $\alpha_{i}$ changes, the problem needs to be solved again.

2. There is no constant and fixed way to find the value of the intended objective function. We need to change the value of $\alpha_{i}$ to arrive at the intended value of the objective function. To do so, we need to solve the problem several times.

It overcome the above shortcomings, we present a GP approach to solve the following problem:

$$
\begin{gathered}
\text { Min } D=d_{1}^{-}-d_{1}^{+}+d_{2}^{-}-d_{2}^{+} \\
\text {s.t. } \mathfrak{R}(Z(\tilde{c}, x))+d_{1}^{-}-d_{1}^{+}=\hat{z} \\
\sum_{i=1}^{m} \alpha_{i}+d_{2}^{-}-d_{2}^{+}=\widehat{m} \\
a_{i} x \leq b_{i}+p_{i}\left(1-\alpha_{i}\right), \quad i=1,2, \ldots, m, \\
\alpha_{i} \geq \alpha_{i}^{D}, 0 \leq \alpha_{i} \leq 1, \quad i=1,2, \ldots, m, \\
d_{1}^{-} \geq 0, d_{1}^{+} \geq 0, d_{2}^{-} \geq 0, d_{2}^{+} \geq 0, \\
x_{j} \geq 0, \quad j=1,2, \ldots, n .
\end{gathered}
$$


We call the above model as Multi-Parametric Linear Goal Programming (MPLGP) problem.

Remark 2. In the above model, the parameters $\hat{z}$ and $\hat{m}$ are the values for objective function and $\sum_{i=1}^{m} \alpha_{i}$ to be given by decision maker.

Remark 3. We will have the two following states after solving the model.

First state: The problem has an optimal result, when the value of objective function gets negative.

Second state: There is no feasible solution, thus the value of the intended objective function of $\hat{z}$ is not appropriate.

Remark 4. It should be noted that if we want to consider a reasonable and acceptable decision about the intended objective function of $\hat{z}$ and value of $\sum_{i=1}^{m} \alpha_{i}$, it is right to solve the first problem in a condition that $\alpha_{i}$ is equal to 1 to have a least acceptable value of objective function $(\hat{z})$.

Then, we replace the $\alpha_{i}$ numbers instated of $\widehat{m}$.

Remark 5. One of the advantages of the above model is that by solving the problem, we will certainly have an optimal solution recognizing a logical limit of $\hat{z}, \widehat{m}, \alpha_{i}^{D}$ selection in order to do sensitivity analysis.

\section{Algorithm 2. Main steps of GP approach for FFLP problem}

Assumption 1: A Fuzzy Flexible Linear Programming (FFLP) problem is given to be solved such as Problem (3). The parameters of the its equivalent model is given by (7) named as MPLP 1, including $\tilde{c}_{j}$, for $j=1,2, \ldots, n$ and $a_{i}, b_{i}, p_{i}, \alpha_{i}^{D}$, for all $i=1,2, \ldots, m$ and $z^{*}$ as a given value by the decision maker for the objective value of problem (10).

Assumption 2: A kind of linear ranking function for ordering of fuzzy parameters is used.

Step 1: Using the given ranking function, obtain the corresponding crisp objective function for the objective function of MPLP1.

Step 2: Obtain the corresponding Multi-Parametric Linear Goal Programming (MPLGP) problem for problem (7) based on (21)

Step3: Solve the MPLGP problem and obtain the optimal value of $\hat{x}^{*}$ and $\hat{\alpha}^{*}$, and finally the optimal value of the objective function as $D^{*}, \hat{Z}^{*}(x)=\mathfrak{R}\left(z\left(\tilde{c}, \hat{x}^{*}\right)\right)$ and $\widehat{W}_{\alpha}^{*}=\sum_{i=1}^{m} \hat{\alpha}_{i}^{*}$.

\section{Numerical study}

In this section, we are going to illustrate the proposed algorithms given in the previous section.

Example 1. Consider the following problem:

$$
\begin{array}{ll}
\text { Max } & Z(\tilde{c}, x)=\tilde{c}_{1} x_{1}+\tilde{c}_{2} x_{2}+\tilde{c}_{3} x_{3}+\tilde{c}_{4} x_{4} \\
\text { s.t. } & x_{1}+x_{2}+x_{3}+x_{4} \preccurlyeq F 15 \\
& 7 x_{1}+5 x_{2}+3 x_{3}+2 x_{4} \preccurlyeq F 80 \\
& 3 x_{1}+4.4 x_{2}+10 x_{3}+15 x_{4} \preccurlyeq^{F} 100 \\
& x_{1} \geq 0, x_{2} \geq 0, x_{3} \geq 0, x_{4} \geq 0,
\end{array}
$$


where the fuzzy coefficients of the objective function are assumed to be $\tilde{c}_{1}=(2,5,1,3), \tilde{c}_{2}=$ $(3,7,1,1), \quad \tilde{c}_{3}=(7,10,1,3), \tilde{c}_{4}=(10,12,2,2)$ and $\quad p_{1}=5, p_{2}=40, p_{3}=30$ are predefined maximum tolerances for $b_{i}(i=1,2,3)$, and $\alpha_{1}^{D}=0.5, \alpha_{2}^{D}=0.5, \alpha_{3}^{D}=0.5$ as the lower bounds of the satisfaction degrees which is desired, for the $i$ th constraint $(i=1,2,3)$, given by the decision maker.

Solution process: The optimal solution of the above FFLP problem are obtained by Algorithm 1 and Algorithm 2.

\subsection{Optimal solution using Algorithm 1}

Here, we are going to obtain the optimal solution of the FFLP problem which given as (22) by Algorithm 1. The main steps of this algorithm are given below.

Step 1: Using the given ranking function (Yager ranking function [6]), we obtain the corresponding crisp objective function for the fuzzy objective function as follows.

$$
\max z(x)=\max \mathfrak{R}(z(\tilde{c}, x))=4 x_{1}+5 x_{2}+9 x_{3}+11 x_{4} .
$$

Step 2: Construct the corresponding MPLP 1 for problem (15) as follows:

$$
\begin{gathered}
\text { Max } \quad Z(x)=4 x_{1}+5 x_{2}+9 x_{3}+11 x_{4} \\
\text { s.t. } \quad x_{1}+x_{2}+x_{3}+x_{4} \leq 15+5\left(1-\alpha_{1}\right) \\
7 x_{1}+5 x_{2}+3 x_{3}+2 x_{4} \leq 80+40\left(1-\alpha_{2}\right) \\
3 x_{1}+4.4 x_{2}+10 x_{3}+15 x_{4} \leq 100+30\left(1-\alpha_{3}\right) \\
0.5 \leq \alpha_{1} \leq 1,0.5 \leq \alpha_{2} \leq 1,0.5 \leq \alpha_{3} \leq 1, x_{1}, x_{2}, x_{3}, x_{4} \geq 0,
\end{gathered}
$$

Step 3: The optimal solution of problem (23) is obtained by Lingo software package as follows:

$$
\begin{gathered}
x_{1}^{*}=8.571429, x_{2}^{*}=0, x_{3}^{*}=8.928571, x_{4}^{*}=0, \alpha_{1}^{*}=0.5, \alpha_{2}^{*}=0.5, \alpha_{3}^{*}=0.5, \\
\text { and } Z^{*}=\Re(z(\tilde{c}, x))=114.6428571 .
\end{gathered}
$$

Step 4: Based on the optimal solution of problem (23) in Step 3, construct the MPLP 2 as follows:

$$
\begin{gathered}
\operatorname{Max} \quad W_{\alpha}=\alpha_{1}+\alpha_{2}+\alpha_{3} \\
\text { s.t. } \quad 4 x_{1}+5 x_{2}+9 x_{3}+11 x_{4} \geq 114.6428571 \\
x_{1}+x_{2}+x_{3}+x_{4} \leq 15+5\left(1-\alpha_{1}\right) \\
7 x_{1}+5 x_{2}+3 x_{3}+2 x_{4} \leq 80+40\left(1-\alpha_{2}\right) \\
3 x_{1}+4.4 x_{2}+10 x_{3}+15 x_{4} \leq 100+30\left(1-\alpha_{3}\right) \\
0.5 \leq \alpha_{1} \leq 1,0.5 \leq \alpha_{2} \leq 1,0.5 \leq \alpha_{3} \leq 1, x_{1}, x_{2}, x_{3}, x_{4} \geq 0,
\end{gathered}
$$

Step 5: The optimal solution of the above problem is founded by Lingo software package as follows:

$$
x_{1}^{* *}=4.047619, x_{2}^{* *}=5.654762, x_{3}^{* *}=7.797619, x_{4}^{* *}=0, \alpha_{1}^{* *}=0.5, \alpha_{2}^{* *}=1, \alpha_{3}^{* *}=0.5,
$$




$$
W_{\alpha}^{* *}=2
$$

Then, the optimal value of the objective function of problem (24) is:

$$
Z^{* *}=\Re(z(\tilde{c}, x))=114.6428571 .
$$

\subsection{Optimal solution using Algorithm 2}

Now, again consider the FFLP problem given by (22). In this part, the problem is solved by Algorithm 2.

Step 1: Using the given ranking function, we obtain the corresponding crisp objective function for the objective function of the problem as follows:

$$
\operatorname{Max} \mathfrak{R}(z(\tilde{c}, x))=\operatorname{Max} z(x)=4 x_{1}+5 x_{2}+9 x_{3}+11 x_{4} .
$$

Note that in this problem, we applied Yager's ranking function [6].

Step 2: Construct the corresponding Multi-Parametric Linear Goal Programming (MPLGP) problem for problem (22) as follows:

$$
\begin{gathered}
\text { Min } D=d_{1}^{-}-d_{1}^{+}+d_{2}^{-}-d_{2}^{+} \\
\text {s.t. } \quad 4 x_{1}+5 x_{2}+9 x_{3}+11 x_{4}-d_{1}^{+}+d_{1}^{-}=99 \\
\alpha_{1}+\alpha_{2}+\alpha_{3}-d_{2}^{+}+d_{2}^{-}=3 \\
x_{1}+x_{2}+x_{3}+x_{4} \leq 15+5\left(1-\alpha_{1}\right) \\
7 x_{1}+5 x_{2}+3 x_{3}+2 x_{4} \leq 80+40\left(1-\alpha_{2}\right) \\
3 x_{1}+4.4 x_{2}+10 x_{3}+15 x_{4} \leq 100+30\left(1-\alpha_{3}\right) \\
0.5 \leq \alpha_{1} \leq 1,0.5 \leq \alpha_{2} \leq 1,0.5 \leq \alpha_{3} \leq 1, \\
x_{1}, x_{2}, x_{3}, x_{4} \geq 0, d_{1}^{-}, d_{1}^{+}, d_{2}^{-}, d_{2}^{+} \geq 0 .
\end{gathered}
$$

Step 3: The optimal solution of the above problem is obtained by the Lingo software package as follows:

$$
\begin{gathered}
\hat{x}_{1}^{*}=4.047619, \hat{x}_{2}^{*}=5.654762, \hat{x}_{3}^{*}=7.797619, \hat{x}_{4}^{*}=0, \hat{\alpha}_{1}^{*}=0.5, \hat{\alpha}_{2}^{*}=1, \hat{\alpha}_{3}^{*}=0.5, d_{1}^{-*}=0, \\
d_{1}^{+^{*}}=15.64286, d_{2}^{-*}=1, d_{2}^{+*}=0, D^{*}=-14.64289, \hat{Z}^{*}(x)=\Re(z(\tilde{c}, x))=114.642857
\end{gathered}
$$

Remark 6. By solving the mentioned FFLP problem using the two different methods, in fact, Algorithm 1 and Algorithm 2, we observed that the proposed optimal solution of problem (25) is actually the optimal solution of problem (24). This result indicates that the proposed method based on the MPLGP model, as given in (21), is more convenient and more practical, since our method can solve the problem merely by a single process directly.

\section{Sensitivity analysis}

In the previous section, we showed that the problem

$$
\begin{array}{cc}
\text { Min } & D=d_{1}^{-}-d_{1}^{+}+d_{2}^{-}-d_{2}^{+} \\
\text {s.t. } & \Re(Z(\tilde{c}, x))+d_{1}^{-}-d_{1}^{+}=\hat{z}
\end{array}
$$




$$
\begin{gathered}
\sum_{i=1}^{m} \alpha_{i}+d_{2}^{-}-d_{2}^{+}=\widehat{m} \\
a_{i} x \leq b_{i}+p_{i}\left(1-\alpha_{i}\right), \quad i=1,2, \ldots, m \\
\alpha_{i} \geq \alpha_{i}^{D}, 0 \leq \alpha_{i} \leq 1, \quad i=1,2, \ldots, m, \\
d_{1}^{-}, d_{1}^{+}, d_{2}^{-}, d_{2}^{+} \geq 0, x_{j} \geq 0, \quad j=1,2, \ldots, n .
\end{gathered}
$$

Is easier to solve in which we directly consider the $\alpha$-cuts of the constraints in the auxiliary objective function. In particular, we saw that our proposed approach could solve the main problem directly by solving just one problem, while the suggested approach by Attari and Nasseri [1], needs to find an optimal solution by solving two problems and in particular, using two phases. Now, we are going to perform a sensitivity analysis of the optimal solution by changing some known parameters of the right-hand-sides such as $\alpha_{i}^{D}, i=1,2, \ldots, m\left(\alpha_{i}^{D}\right.$ : Lower bound of the $\alpha$-cut for the $i$ th constraint).

5.1. In the first evaluation, we consider the amount of $\alpha_{1}^{D}$ and $\alpha_{2}^{D}$ be equal to one and also consider $\alpha_{3}^{D} \in[0.1,1]$. Then, by solving the corresponding MPLGP problem (26) for problem (23),

\begin{tabular}{|c|c|c|c|c|c|c|c|c|c|c|}
\hline$\alpha_{3}^{D}$ & 0.1 & 0.2 & 0.3 & 0.4 & 0.5 & 0.6 & 0.7 & 0.8 & 0.9 & 1 \\
\hline$Z$ & $\begin{array}{l}\vec{D} \\
\infty \\
\dot{\vec{u}} \\
\vec{D}\end{array}$ & 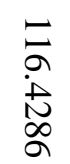 & $\begin{array}{l}\vec{\nabla} \\
\stackrel{\vec{N}}{\infty} \\
\dot{\infty} \\
\dot{y}\end{array}$ & 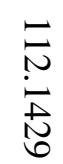 & $\Xi$ & $\begin{array}{l}\vec{\jmath} \\
\infty \\
\infty \\
y \\
\forall\end{array}$ & 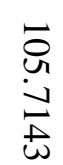 & $\begin{array}{l}\vec{o} \\
\text { w } \\
\text { in } \\
\vec{\phi}\end{array}$ & $\begin{array}{l}\overrightarrow{0} \\
\stackrel{\vec{A}}{+} \\
\stackrel{\infty}{\infty} \\
a\end{array}$ & $\begin{array}{l}0 \\
\text { N } \\
\infty \\
\text { un } \\
\text { un }\end{array}$ \\
\hline
\end{tabular}
we obtain the optimal solution and the optimal value of the objective function $\Re(z(\tilde{c}, x))$.

Table 1. Objective function value for problem (25) based on the amount of $\boldsymbol{\alpha}_{3}^{\boldsymbol{D}}$ from 0.1 to 1

Table 1 shows that various obtained values of the objective function using various values of $\alpha_{3}^{D}$ from 0.1 to 1 . We see that by increasing the amount of parameter $\alpha_{3}^{D}$, the objective function value decreases. 


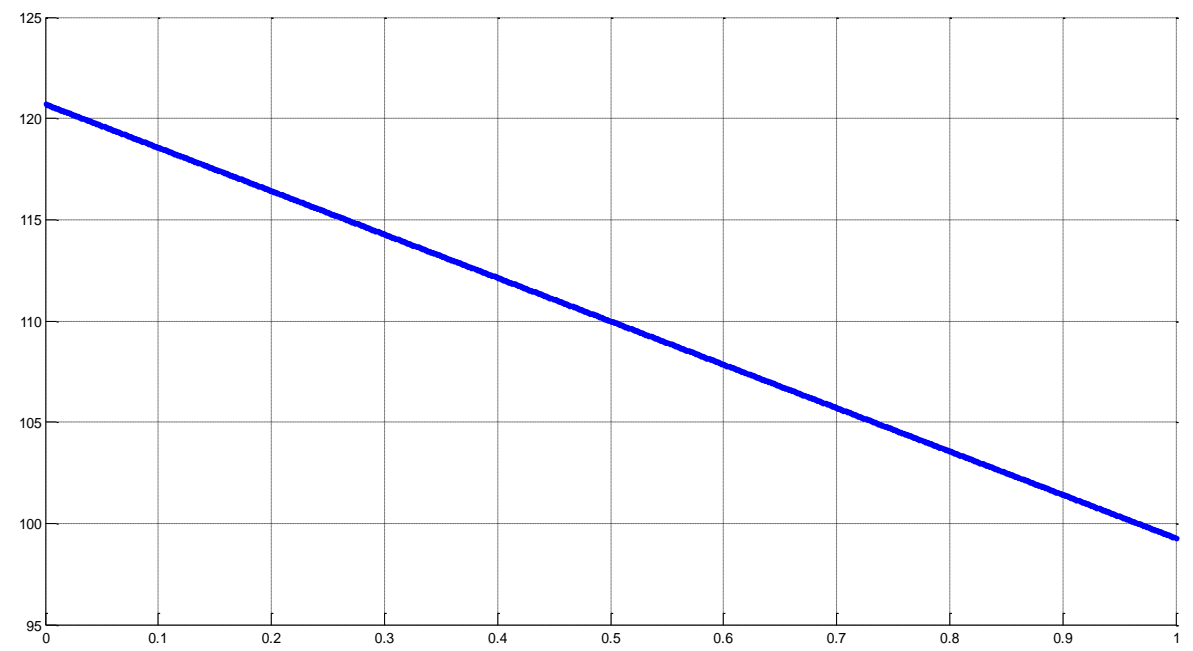

Figure 1: Variation of the objective function with the given results in Table 1

Figure 1 shows that the variation of the objective function based on the variation of the parameter $\alpha_{3}^{D} \in[0.1,1]$. We see that the minimum and the maximum values of the objective function are respectively 99.2857 and 118.5714 .

5.2. In the second examination, we consider the variation of the objective function values based on the variation of the parameter $\alpha_{2}^{D}$ which is allowed to change from 0.1 to 1 with a fixed value, or $\alpha_{1}^{D}$ and $\alpha_{3}^{D}$ which is considered by $\alpha_{1}^{D}=\alpha_{3}^{D}=1$. Various values of the objective function for the MPLGP problem associated with problem (25) are reported in Table 2.

Table 2. Objective function value for problem (25) based on

\begin{tabular}{|c|c|c|c|c|c|c|c|c|c|c|}
\hline \multicolumn{11}{|c|}{ the amount of $\boldsymbol{\alpha}_{2}^{\boldsymbol{D}}$ from 0.1 to 1} \\
\hline$\alpha_{2}^{D}$ & 0.1 & 0.2 & 0.3 & 0.4 & 0.5 & 0.6 & 0.7 & 0.8 & 0.9 & 1 \\
\hline$Z$ & $\begin{array}{l}8 \\
\text { v } \\
\infty \\
\text { y } \\
\text { v }\end{array}$ & $\begin{array}{l}0 \\
\text { D } \\
\infty \\
\text { y } \\
ચ\end{array}$ & $\begin{array}{l}\text { o } \\
\text { N } \\
\infty \\
\text { y }\end{array}$ & 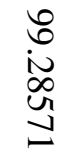 & $\begin{array}{l}\text { o } \\
\text { in } \\
\infty \\
\text { y }\end{array}$ & $\begin{array}{l}\text { D } \\
\text { i } \\
\infty \\
\text { ચ }\end{array}$ & $\begin{array}{l}0 \\
0 \\
\text { i } \\
\text { y } \\
\text { v }\end{array}$ & 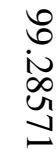 & $\begin{array}{l}8 \\
\dot{0} \\
\infty \\
\text { y } \\
\forall\end{array}$ & 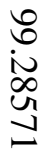 \\
\hline
\end{tabular}

Clearly, the obtained results show that there is not improvement on the objective function value based on the variation of the parameter $\alpha_{2}^{D}$. Thus, we realize that the increasing the resource level for the second constraint is not effective in improving the optimal value of the objective function. 


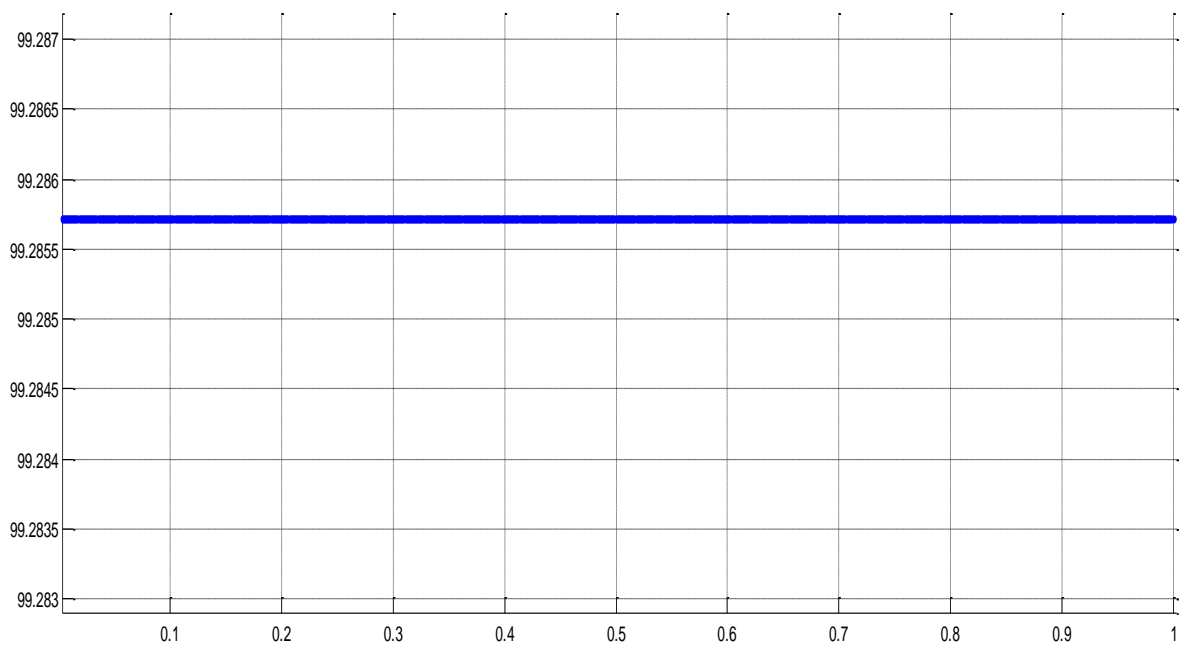

Figure 2: Variation of the objective function with the given results in Table 2

5.3. In the third examination, we explore the variation of the objective function values based on the variation in the first lower bound of the satisfaction level that is $\alpha_{1}^{D}$ with a fixed values for the second and the third levels using $\alpha_{2}^{D}=\alpha_{3}^{D}=1$. The obtained numerical results from solving the MPLGP problem associated with problem (25) are given in Table 3.

Table 3. Objective function value for problem (25) based on the amount of $\boldsymbol{\alpha}_{1}^{D}$ from 0.1 to 1

\begin{tabular}{|c|c|c|c|c|c|c|c|c|c|c|}
\hline$\alpha_{1}^{D}$ & 0.1 & 0.2 & 0.3 & 0.4 & 0.5 & 0.6 & 0.7 & 0.8 & 0.9 & 1 \\
\hline$Z$ & $\begin{array}{l}\stackrel{\vec{P}}{+} \\
\stackrel{\vec{\omega}}{\stackrel{p}{+}}\end{array}$ & $\begin{array}{l}\stackrel{\vec{P}}{+} \\
\stackrel{\vec{\omega}}{\stackrel{p}{+}}\end{array}$ & $\begin{array}{l}\vec{\varnothing} \\
\stackrel{+}{\dot{\omega}} \\
\stackrel{p}{+}\end{array}$ & $\begin{array}{l}\vec{\triangleright} \\
\stackrel{+}{\dot{\omega}} \\
\stackrel{p}{+}\end{array}$ & $\begin{array}{l}\overrightarrow{0} \\
\text { ర } \\
\text { No } \\
\infty \\
0\end{array}$ & i & 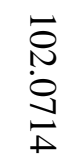 & 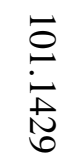 & $\begin{array}{l}\overrightarrow{8} \\
\stackrel{8}{\sim} \\
\stackrel{+}{\omega}\end{array}$ & 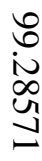 \\
\hline
\end{tabular}

In our examination, we see that increasing the resource level for the first constraint is strongly effective when the degree satisfaction goes beyond 0.5 . Hence, any decrease in the degree of satisfaction of the first constraint from 0.5 to zero will be concluded a great improvement on the optimal value of the objective function.

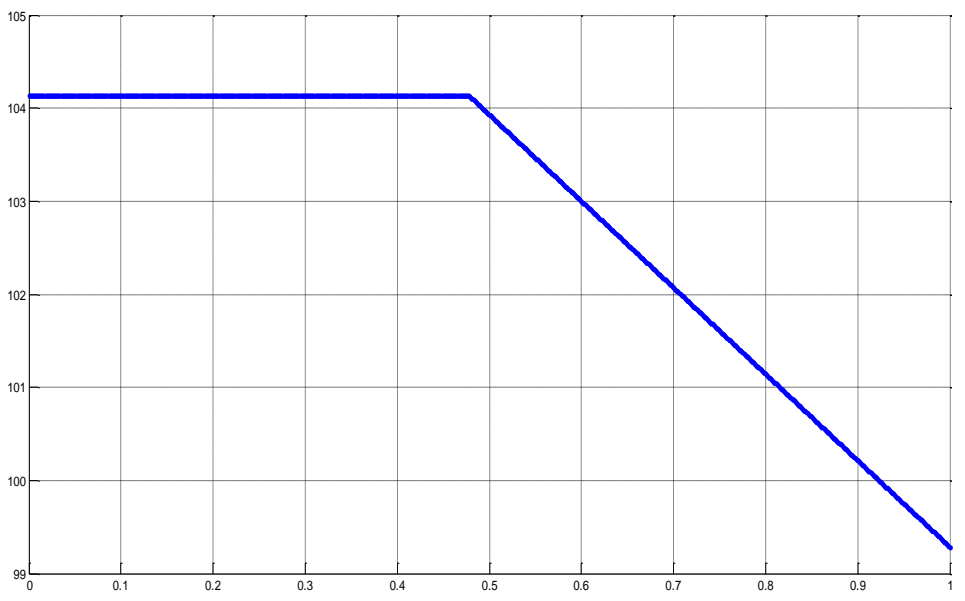


Figure 3: Variation of the objective function with the given results in Table3

Figure 3 shows the mentioned result, graphically.

Remark 7. The above numerical examination shows that variation on the resource level of the second constraint is not effective for improve the current optimal value of the objective function. However, the objective function value is sensitive when the available resources for the first and the third constraints change.

The above remark leads us to focus on a new examination to explore variation of the optimal value of the objective function with respect to variations of the first and the third available resource levels simultaneously. Table 4 gives the result of this examination.

\begin{tabular}{|c|c|c|c|c|c|c|c|c|c|c|}
\hline$\mapsto$ & م) & ○ & $\stackrel{\ominus}{ }$ & $\begin{array}{l}\circ \\
\text { ă }\end{array}$ & $\begin{array}{l}\circ \\
\text { ir }\end{array}$ & $\stackrel{\circ}{\circ}$ & $\stackrel{\circ}{\dot{\omega}}$ & $\stackrel{\circ}{i}$ & $\stackrel{\circ}{\ominus}$ & $\left.{ }^{\infty}\right) / 8$ \\
\hline 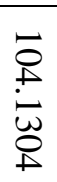 & $\begin{array}{l}\vec{\Xi} \\
\text { uे } \\
\text { à }\end{array}$ & 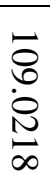 & $\begin{array}{l}\text { 三 } \\
\stackrel{\vec{D}}{\vec{a}} \\
\vec{D}\end{array}$ & $\begin{array}{l}\text { ప } \\
\underset{\sigma}{\sigma} \\
\underline{\omega}\end{array}$ & 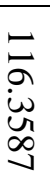 & $\begin{array}{l}\varpi \\
\infty \\
\infty \\
\infty \\
\stackrel{\infty}{\omega}\end{array}$ & $\frac{\bar{N}}{\stackrel{\sim}{u}}$ & $\begin{array}{l}\vec{N} \\
\dot{w} \\
\hat{d} \\
\text { Ŭ }\end{array}$ & $\begin{array}{l}\bar{N} \\
\Omega \\
\text { N } \\
\stackrel{ \pm}{\omega}\end{array}$ & $\stackrel{\circ}{\bullet}$ \\
\hline $\begin{array}{l}\vec{\delta} \\
\stackrel{\phi}{\dot{\omega}} \\
\stackrel{\phi}{+}\end{array}$ & $\begin{array}{l}\overrightarrow{8} \\
\text { ù } \\
\text { à }\end{array}$ & $\begin{array}{l}\overrightarrow{0} \\
0 \\
\stackrel{0}{0} \\
\stackrel{\infty}{\infty}\end{array}$ & $\begin{array}{l}\text { 三 } \\
\stackrel{\vec{A}}{\vec{a}} \\
\vec{D}\end{array}$ & $\begin{array}{l}\text { ప } \\
\underline{\sigma} \\
\underline{\omega}\end{array}$ & 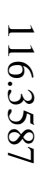 & $\begin{array}{l}\varpi \\
\infty \\
\infty \\
\infty \\
\dot{\omega}\end{array}$ & $\frac{\bar{N}}{\stackrel{\sim}{u}}$ & 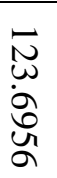 & $\overrightarrow{\tilde{a}}$ & $\stackrel{\circ}{\sim}$ \\
\hline $\begin{array}{l}\vec{D} \\
\stackrel{+}{\dot{\omega}} \\
\stackrel{\phi}{+}\end{array}$ & $\begin{array}{l}\overrightarrow{8} \\
\text { ù } \\
\text { à }\end{array}$ & 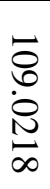 & $\begin{array}{l}\text { 三 } \\
\dot{\vec{A}} \\
\vec{a}\end{array}$ & $\begin{array}{l}\vec{\omega} \\
\dot{\omega} \\
\vec{\omega}\end{array}$ & $\begin{array}{l}\overrightarrow{\vec{\sigma}} \\
\dot{a} \\
\dot{w} \\
\dot{\infty} \\
\dot{v}\end{array}$ & $\begin{array}{l}\text { ప } \\
\infty \\
\dot{+} \\
\text { N }\end{array}$ & $\begin{array}{l}\vec{N} \\
\stackrel{0}{\sim} \\
\text { D } \\
\text { ù }\end{array}$ & $\begin{array}{l}\vec{N} \\
\text { No } \\
\text { N } \\
\infty\end{array}$ & 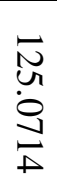 & $\stackrel{\circ}{\dot{\omega}}$ \\
\hline 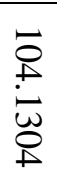 & $\begin{array}{l}\overrightarrow{8} \\
\text { ù } \\
\text { ă }\end{array}$ & $\begin{array}{l}\overrightarrow{8} \\
\dot{0} \\
\dot{\infty} \\
\stackrel{\infty}{\infty}\end{array}$ & $\begin{array}{l}\text { 三 } \\
\text { i } \\
\dot{y}\end{array}$ & $\begin{array}{l}\vec{\omega} \\
\dot{\omega} \\
\stackrel{\Delta}{N} \\
a \\
a\end{array}$ & $\begin{array}{l}\overrightarrow{\vec{u}} \\
\dot{u} \\
\vec{v} \\
\vec{A}\end{array}$ & 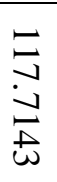 & $\begin{array}{l}\vec{\varpi} \\
\dot{\infty} \\
\text { uy } \\
ચ\end{array}$ & $\stackrel{N}{N}$ & $\frac{\vec{N}}{\stackrel{+}{+}}$ & if \\
\hline
\end{tabular}




\begin{tabular}{|c|c|c|c|c|c|c|c|c|c|c|}
\hline 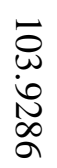 & 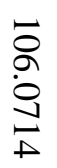 & 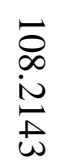 & 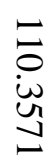 & $\underset{N}{\tilde{N}}$ & 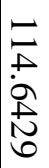 & $\begin{array}{l}\text { 三人 } \\
\dot{y} \\
\text { un }\end{array}$ & $\begin{array}{l}\varpi \\
\infty \\
\dot{0} \\
\text { 心 } \\
\sigma\end{array}$ & 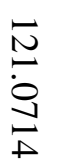 & 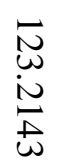 & ن \\
\hline$\stackrel{\vec{\omega}}{\mathrm{\omega}}$ & 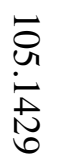 & $\begin{array}{l}\text { 어 } \\
\text { in } \\
\infty \\
\text { y }\end{array}$ & $\begin{array}{l}\overrightarrow{0} \\
\stackrel{0}{0} \\
\stackrel{+}{N} \\
\infty\end{array}$ & $\underset{\vec{D}}{\overrightarrow{\vec{v}}}$ & $\begin{array}{l}\vec{\omega} \\
\overrightarrow{0} \\
\stackrel{\vec{\omega}}{\omega}\end{array}$ & $\begin{array}{l}\text { Е } \\
\dot{\infty} \\
\dot{y} \\
y\end{array}$ & $\vec{\infty}$ & $\begin{array}{l}\vec{N} \\
\stackrel{\vec{D}}{\mathbb{J}} \\
\stackrel{0}{0}\end{array}$ & $\begin{array}{l}\bar{N} \\
N \\
N \\
\infty \\
\text { y }\end{array}$ & $\begin{array}{l}\circ \\
\text { ă }\end{array}$ \\
\hline i & $\begin{array}{l}\vec{D} \\
+\underset{+}{N} \\
\stackrel{\vec{t}}{\omega}\end{array}$ & 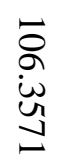 & $\begin{array}{l}\overrightarrow{0} \\
\infty \\
\text { in }\end{array}$ & 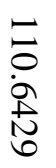 & 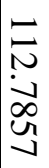 & 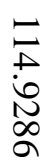 & 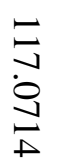 & $\begin{array}{l}\vec{\sigma} \\
\stackrel{\vec{N}}{\vec{t}} \\
\stackrel{\omega}{\omega}\end{array}$ & 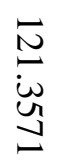 & $\stackrel{0}{V}$ \\
\hline 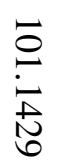 & 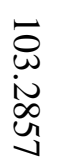 & $\begin{array}{l}\overrightarrow{0} \\
\text { ur } \\
+ \\
\text { N } \\
\infty \\
a\end{array}$ & $\begin{array}{l}\overrightarrow{0} \\
\text { in } \\
\underset{D}{\vec{D}}\end{array}$ & 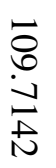 & $\begin{array}{l}\Xi \\
\dot{\infty} \\
\dot{y} \\
\text { J }\end{array}$ & $\vec{\nexists}$ & 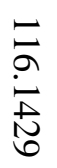 & 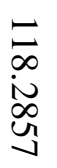 & $\begin{array}{l}\vec{N} \\
\stackrel{0}{0} \\
\stackrel{+}{N} \\
\infty \\
\sigma\end{array}$ & $\begin{array}{l}\text { ○ } \\
\infty\end{array}$ \\
\hline 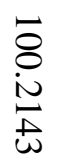 & 요 & 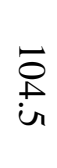 & 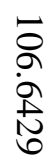 & 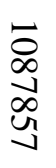 & $\begin{array}{l}= \\
0 \\
i 0 \\
N \\
\infty \\
\sigma\end{array}$ & $\begin{array}{l}\text { Е } \\
\stackrel{\omega}{\mathcal{U}} \\
\underset{\Delta}{\Delta}\end{array}$ & 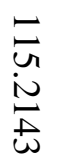 & $\begin{array}{l}\vec{\Xi} \\
\underset{w}{w} \\
\vec{v}\end{array}$ & $\begin{array}{l}\text { च } \\
\text { in }_{1}\end{array}$ & Q \\
\hline $\begin{array}{l}0 \\
\text { i } \\
\infty \\
\text { y } \\
ચ\end{array}$ & $\overline{0}$ & $\begin{array}{l}\overrightarrow{0} \\
\dot{w} \\
\dot{u} \\
\vec{D}\end{array}$ & $\begin{array}{l}\overrightarrow{0} \\
\dot{\omega} \\
\dot{\vec{\nu}} \\
\stackrel{\vec{\omega}}{ }\end{array}$ & $\begin{array}{l}\overrightarrow{0} \\
\dot{y} \\
\infty \\
y \\
y\end{array}$ & $\bar{\sigma}$ & 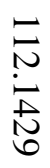 & 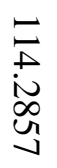 & $\begin{array}{l}\vec{a} \\
\underset{\mathbb{A}}{\infty} \\
\stackrel{\infty}{a}\end{array}$ & $\begin{array}{l}\vec{\infty} \\
\dot{\infty} \\
\vec{\Delta}\end{array}$ & $\mapsto$ \\
\hline
\end{tabular}

Table 4 gives various values of the objective function of problem (25) using the variation from 0.1 to 1 for $\alpha_{1}^{D}$ and $\alpha_{3}^{D}$.

The results in Table 4 show that the optimal value of the objective function with respect to the parameter $\alpha_{3}^{D}$ is more sensitive as compared to the parameter $\alpha_{1}^{D}$.

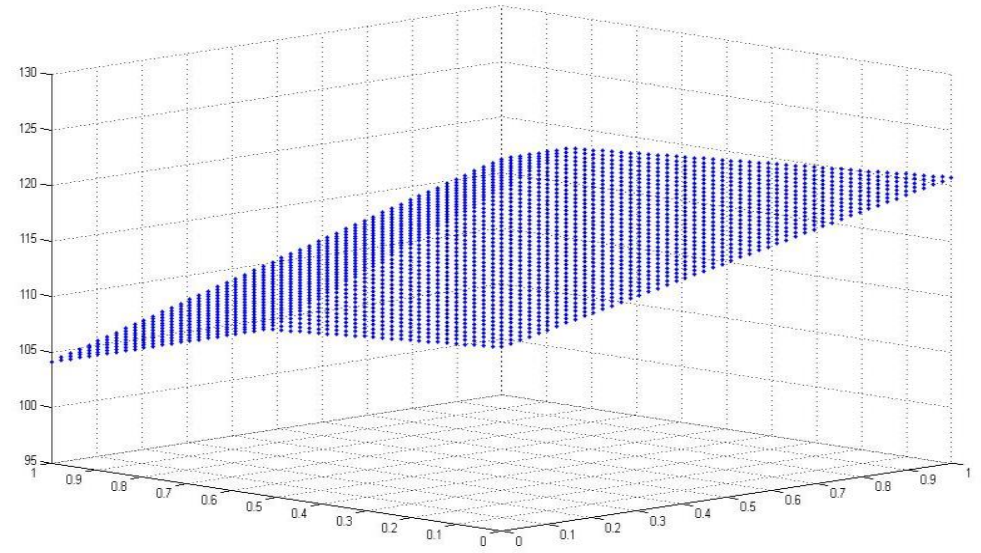

Figure 4: The variation value of the objective function corresponding to the given results in Table 4 
5.5 Now, we are at a place to give more discussions of sensitivity analysis for a decision maker to improve the satisfaction level with variation upon all parameters.

Now, assume that the decision maker would like to make an optimal decision for the MPLGP problem associated as problem (23), while the summation value of the satisfaction level of all the constraints are to be equal or bigger than a desirable value. In fact, this case will be an important model of the MPLGP problem. To this aim, we are going to solve two numerical examples.

Example 5.1. Consider problem (25) with the new conditions as follows:

$$
\alpha_{1} \geq 0.4, \alpha_{2} \geq 0.4, \alpha_{3} \geq 0.4, \alpha_{1}+\alpha_{2}+\alpha_{3} \geq 2.1 \text {. }
$$

Thus, we have the following problem to solve:

$$
\begin{gathered}
\text { Min } D=d_{1}^{-}-d_{1}^{+}+d_{2}^{-}-d_{2}^{+} \\
\text {s.t. } \quad 4 x_{1}+5 x_{2}+9 x_{3}+11 x_{4}-d_{1}^{+}+d_{1}^{-}=99 \\
\alpha_{1}+\alpha_{2}+\alpha_{3}-d_{2}^{+}+d_{2}^{-}=3 \\
x_{1}+x_{2}+x_{3}+x_{4} \leq 15+5\left(1-\alpha_{1}\right) \\
7 x_{1}+5 x_{2}+3 x_{3}+2 x_{4} \leq 80+40\left(1-\alpha_{2}\right) \\
3 x_{1}+4.4 x_{2}+10 x_{3}+15 x_{4} \leq 100+30\left(1-\alpha_{3}\right) \\
\alpha_{1}+\alpha_{2}+\alpha_{3} \geq 2.1 \\
0.4 \leq \alpha_{1} \leq 1,0.4 \leq \alpha_{2} \leq 1,0.4 \leq \alpha_{3} \leq 1 \\
d_{1}^{-} \geq 0, d_{1}^{+} \geq 0, d_{2}^{-} \geq 0, d_{2}^{+} \geq 0 \\
x_{1} \geq 0, x_{2} \geq 0, x_{3} \geq 0, x_{4} \geq 0 .
\end{gathered}
$$

Solving the above model, we get the optimal solution as follows:

$$
\hat{\alpha}_{1}^{*}=0.7, \hat{\alpha}_{2}^{*}=1, \hat{\alpha}_{3}^{*}=0.4, \hat{Z}^{*}=\Re\left(z\left(\tilde{c}, \hat{x}^{*}\right)\right)=114.642857, \widehat{W}_{i}^{*}=\sum_{i=1}^{3} \hat{\alpha}_{i}^{*}=2.1 .
$$

The obtained results show that the suggested model can obtain an optimal solution having the same value for the objective function of problem (25) with a higher degree for the summation of the parameters $\alpha_{i}(i=1,2,3)$. In fact, our approach can obtain the optimal solution of the main problem by a simpler process, being more comfortable of the decision maker.

Example 5.2. Consider problem (25) with the following new conditions:

$$
\alpha_{1} \geq 0.4, \alpha_{2} \geq 0.5, \alpha_{3} \geq 0.3, \alpha_{1}+\alpha_{2}+\alpha_{3} \geq 2.2 .
$$

Then, problem (25) is changed to:

$$
\begin{gathered}
\text { Min } D=d_{1}^{-}-d_{1}^{+}+d_{2}^{-}-d_{2}^{+} \\
\text {s.t. } \quad 4 x_{1}+5 x_{2}+9 x_{3}+11 x_{4}-d_{1}^{+}+d_{1}^{-}=99 \\
\alpha_{1}+\alpha_{2}+\alpha_{3}-d_{2}^{+}+d_{2}^{-}=3 \\
x_{1}+x_{2}+x_{3}+x_{4} \leq 15+5\left(1-\alpha_{1}\right) \\
7 x_{1}+5 x_{2}+3 x_{3}+2 x_{4} \leq 80+40\left(1-\alpha_{2}\right) \\
3 x_{1}+4.4 x_{2}+10 x_{3}+15 x_{4} \leq 100+30\left(1-\alpha_{3}\right) \\
x_{1} \geq 0, x_{2} \geq 0, x_{3} \geq 0, x_{4} \geq 0 \\
0.4 \leq \alpha_{1} \leq 1,0.4 \leq \alpha_{2} \leq 1,0.4 \leq \alpha_{3} \leq 1, \\
\alpha_{1}+\alpha_{2}+\alpha_{3} \geq 2.1 \\
d_{1}^{-} \geq 0, d_{1}^{+} \geq 0, d_{2}^{-} \geq 0, d_{2}^{+} \geq 0 .
\end{gathered}
$$


The optimal solution of the main problem will be obtained by solving the above model as follows:

$$
\hat{\alpha}_{1}^{*}=0.9, \widehat{\alpha}_{2}^{*}=1, \hat{\alpha}_{3}^{*}=0.3, \widehat{Z}^{*}(x)=\max \mathfrak{R}\left(z\left(\tilde{c}, \hat{x}^{*}\right)\right)=115.2143
$$

We see that the current model helps us to obtain an optimal solution for the main problem in the following two senses:

(a) The last optimal value obtained by Attari and Nasseri [1] is less than the obtained optimal value by our approach for the main problem.

(b) Unlike the optimal value obtained by Attari and Nasseri [1], our suggested approach obtained a better solution with a higher satisfaction level. In fact, in Attari and Nasseri's solution, the obtained value for the objective unction is 114.642857 with $W_{\alpha}^{* *}=\alpha_{1}^{* *}+\alpha_{2}^{* *}+\alpha_{3}^{* *}=2$, while we obtained the optimal value to be 115.2143, bigger than the optimal solution obtained by Attari and Nasseri [1], and with the general satisfaction level of $\widehat{W}_{\alpha}^{*}=\sum_{i=1}^{3} \hat{\alpha}_{i}^{*}=2.2$.

\section{Case study}

\subsection{The problem}

A manufacturing company desires to maximize the total profit from producing four products $(P M C)_{1},(P M C)_{2},(P M C)_{3}$ and $(P M C)_{4}$ utilizing three different materials $M_{1}, M_{2}$ and $M_{3}$. The required information for producing each product is given in Tables 5 and 6 . The company is to figure out how many units of products $(P M C)_{1},(P M C)_{2},(P M C)_{3}$ and $(P M C)_{4}$ should be produced to maximize the total profit.

Table 5: Required information for four different products studied in manufacturing company

\begin{tabular}{|c|c|c|c|c|}
\hline The required information of the items & $\begin{array}{c}\text { First } \\
\text { product }\end{array}$ & $\begin{array}{l}\text { Second } \\
\text { product }\end{array}$ & $\begin{array}{l}\text { Third } \\
\text { product }\end{array}$ & $\begin{array}{l}\text { Fourth } \\
\text { product }\end{array}$ \\
\hline $\begin{array}{l}\text { The required amount of material } M_{1} \text { for } \\
\text { producing one unit }\end{array}$ & 4.3 & 8 & 5.7 & 12 \\
\hline $\begin{array}{l}\text { The required amount of material } M_{2} \text { for } \\
\text { producing one unit }\end{array}$ & 10 & 3.5 & 7 & 6.5 \\
\hline $\begin{array}{l}\text { The required amount of material } M_{3} \text { for } \\
\text { producing one unit }\end{array}$ & 13 & 15.2 & 4 & 9.1 \\
\hline $\begin{array}{l}\text { The number of used workers (person- } \\
\text { daytime) for producing one unit }\end{array}$ & 11 & 7 & 14 & 10 \\
\hline The gross benefit in producing one unit & 14 & 19 & 15 & 21 \\
\hline The amount of lower bound & 10 & 17 & 12 & 15 \\
\hline The amount of upper bound & 100 & 100 & 100 & 100 \\
\hline
\end{tabular}




\begin{tabular}{|c|c|c|c|c|}
\hline \multicolumn{5}{|c|}{ Table 6: Available resources and acceptable tolerances } \\
\hline The limitations of items & $\begin{array}{c}\text { Material } \\
M_{1}\end{array}$ & $\begin{array}{c}\text { Material } \\
M_{2}\end{array}$ & $\begin{array}{c}\text { Material } \\
M_{3}\end{array}$ & human force \\
\hline $\begin{array}{c}\text { The of lower bound available } \\
\text { resources }\end{array}$ & 1200 & 2300 & 1700 & 4000 \\
\hline $\begin{array}{c}\text { The assumed tolerances for } \\
\text { resources }\end{array}$ & 350 & 200 & 400 & 800 \\
\hline
\end{tabular}

Now, based on the given data in tables 5 and 6, we present and solve a model to determine values of the four products $(P M C)_{1},(P M C)_{2},(P M C)_{3}$ and $(P M C)_{4}$.

\subsection{Formulation of the problem}

\section{Decision variables:}

$x_{1}$ : Amount for the first product

$x_{2}$ : Amount for the second product

$x_{3}$ : Amount for the third product

$x_{4}$ : Amount for the fourth product

\section{Parameters:}

$a_{1 j}$ : Amount of material $M_{1}$ for producing one unit of $j$ th product $(j=1,2,3,4)$,

$a_{2 j}$ : Amount of material $M_{2}$ for producing one unit of $j$ th product $(j=1,2,3,4)$,

$a_{3 j}$ : Amount of material $M_{3}$ for producing one unit of $j$ th product $(j=1,2,3,4)$

$a_{4 j}$ : Number of used workers (person-daytime) for producing one unit of $j$ th product $(j=1,2,3,4)$

$l_{j}$ : Lower bound for the $j$ th product $(j=1,2,3,4)$

$u_{j}$ : Upper bound for the $j$ th product $(j=1,2,3,4)$

$b_{1}$ : Upper bound for available resources of material $M_{1}$

$b_{2}$ : Upper bound for available resources of material $M_{2}$

$b_{3}$ : Upper bound for available resources of material $M_{3}$

$b_{4}$ : Upper bound for available resources of workers

$p_{1}$ : Amount of assumed tolerance for resource material $M_{1}$

$p_{2}$ : Amount of assumed tolerance for resource material $M_{2}$

$p_{3}$ : Amount of assumed tolerance for resource material $M_{3}$ 
$p_{4}$ : Amount of assumed tolerance for resource workers

$c_{j}$ : The Gross benefit for $j$ th product in one unit $(j=1,2,3,4)$

\section{Objective function:}

The objective function considers the maximum value of the gross benefit of producing four products. Therefore, the objective function is

\section{Constraints:}

$$
\max \quad Z(x)=\sum_{j=1}^{4} c_{j} x_{j} .
$$

Examining the available information, six types of constraint are identified:

1. Constraint corresponding to the amount of used material:

$$
M_{1}: \sum_{j=1}^{4} a_{1 j} x_{j} \prec^{F} b_{1}
$$

2 Constraint corresponding to the amount of used material:

$$
M_{2}: \sum_{j=1}^{4} a_{2 j} x_{j} \prec^{F} b_{2}
$$

3. Constraint corresponding to the amount of used material:

$$
M_{3}: \sum_{j=1}^{4} a_{3 j} x_{j} \prec^{F} b_{3}
$$

4. Constraint corresponding to the number of used workers:

$$
M_{4}: \sum_{j=1}^{4} a_{4 j} x_{j} \prec^{F} b_{4}
$$

5. Constraint corresponding to the lower bound for the $j$ th product:

$$
x_{j} \geq l_{j}(j=1,2,3,4)
$$

6. Constraint corresponding to the lower bound for the $j$ th product:

$$
x_{j} \leq u_{j}(j=1,2,3,4)
$$

\subsection{Mathematical model for FFLP problem}

The mathematical formulation of the case study is now given as follows:

$$
\begin{array}{cc}
\text { Max } & Z(x)=25 x_{1}+27 x_{2}+30 x_{3}+28 x_{4} \\
\text { s.t. } & 4.3 x_{1}+8 x_{2}+5.7 x_{3}+12 x_{4} \prec^{F} 1200 \\
& 10 x_{1}+3.5 x_{2}+7 x_{3}+5 x_{4} \prec^{F} 2300 \\
13 x_{1}+15.2 x_{2}+4 x_{3}+9.1 x_{4}{ }^{F} 1700 \\
11 x_{1}+7 x_{2}+14 x_{3}+10 x_{4} \prec^{F} 4000 \\
10 \leq x_{1} \leq 100, \\
17 \leq x_{2} \leq 100, \\
12 \leq x_{3} \leq 100, \\
5 \leq x_{4} \leq 100 \\
x_{1} \geq 0, x_{2} \geq 0, x_{3} \geq 0, x_{4} \geq 0 .
\end{array}
$$

Clearly, we see that the current model is in the form of an FFLP problem as given by (3). We solve this problem in the next subsection. Note that, since in the above model the objective coefficient appear in the crisp sense, Step 1 of both algorithms (Algorithm 1 and Algorithm 2) should omitted, and therefore, we will continue from Step 2.

\subsection{Solution method}


Here, we are going to first obtain the optimal solution of the FFLP problem as given by (26) using Algorithm 1. The main steps of this algorithm is given nest.

Step 2: Obtain the corresponding MPLP 1 for problem (29) as follows:

$$
\begin{array}{cc}
\text { Max } & Z(x)=25 x_{1}+27 x_{2}+30 x_{3}+28 x_{4} \\
\text { s.t. } & 4.3 x_{1}+8 x_{2}+5.7 x_{3}+12 x_{4} \leq 1200+350\left(1-\alpha_{1}\right) \\
10 x_{1}+3.5 x_{2}+7 x_{3}+5 x_{4} \leq 2300+200\left(1-\alpha_{2}\right) \\
13 x_{1}+15.2 x_{2}+4 x_{3}+9.1 x_{4} \leq 1700+400\left(1-\alpha_{3}\right) \\
11 x_{1}+7 x_{2}+14 x_{3}+10 x_{4} \leq 4000+800\left(1-\alpha_{4}\right) \\
10 \leq x_{1} \leq 100 \\
17 \leq x_{2} \leq 100 \\
12 \leq x_{3} \leq 100 \\
5 \leq x_{4} \leq 100 \\
0.4 \leq \alpha_{1} \leq 1 \\
0.4 \leq \alpha_{2} \leq 1 \\
0.4 \leq \alpha_{3} \leq 1 \\
0.4 \leq \alpha_{4} \leq 1 \\
\geq 0, x_{2} \geq 0, x_{3} \geq 0, x_{4} \geq 0 .
\end{array}
$$

Step 3: The optimal solution of model (23) is achieved by means of the Lingo software package:

$$
\begin{gathered}
x_{1}^{*}=76.77590, x_{2}^{*}=17, x_{3}^{*}=100, x_{4}^{*}=31.15530, \alpha_{1}^{*}=0.4, \alpha_{2}^{*}=0.4, \alpha_{3}^{*}=0.4, \\
Z^{*}=6250.746 .
\end{gathered}
$$

Step 4: Based on the optimal solution of problem (27) in Step 2, we obtain the MPLP 2 problem as follows:

$$
\begin{aligned}
& \text { Max } \quad \widehat{W}_{\alpha}=\alpha_{1}+\alpha_{2}+\alpha_{3}+\alpha_{4} \\
& \text { s.t. } 25 x_{1}+27 x_{2}+30 x_{3}+28 x_{4} \geq 6250.746 \\
& 4.3 x_{1}+8 x_{2}+5.7 x_{3}+12 x_{4} \leq 1200+350\left(1-\alpha_{1}\right) \\
& 10 x_{1}+3.5 x_{2}+7 x_{3}+5 x_{4} \leq 2300+200\left(1-\alpha_{2}\right) \\
& 13 x_{1}+15.2 x_{2}+4 x_{3}+9.1 x_{4} \leq 1700+400\left(1-\alpha_{3}\right) \\
& 11 x_{1}+7 x_{2}+14 x_{3}+10 x_{4} \leq 4000+800\left(1-\alpha_{4}\right) \\
& 10 \leq x_{1} \leq 100 \\
& 17 \leq x_{2} \leq 100 \\
& 12 \leq x_{3} \leq 100 \\
& 5 \leq x_{4} \leq 100 \\
& 0.4 \leq \alpha_{1} \leq 1, \\
& 0.4 \leq \alpha_{2} \leq 1, \\
& 0.4 \leq \alpha_{3} \leq 1, \\
& 0.4 \leq \alpha_{4} \leq 1, \\
& x_{1} \geq 0, x_{2} \geq 0, x_{3} \geq 0, x_{4} \geq 0 .
\end{aligned}
$$

Step 5: The optimal solution of the above problem is achieved by means of Lingo software package: 


$$
\begin{gathered}
x_{1}^{* *}=76.77591, x_{2}^{* *}=17, x_{3}^{* *}=100, x_{4}^{* *}=31.15530, \alpha_{1}^{* *}=0.4, \alpha_{2}^{* *}=1, \alpha_{3}^{* *}=0.4, \\
W_{\alpha}^{* *}=2.8 .
\end{gathered}
$$

Thus, the optimal value of the objective function of problem (29) is

$$
Z^{* *}=6250.746 .
$$

Next, we solve the current FFLP problem using Algorithm 2.

Step 2: Construct the corresponding multi parametric linear goal programming (MPLGP) model for problem (26).

Step 3: The optimal solution of problem (26) is found to be

$$
\begin{gathered}
x_{1}^{*}=76.77590, x_{2}^{*}=17, x_{3}^{*}=100, x_{4}^{*}=31.15530, \\
Z^{*}=6250.746, \\
\alpha_{1}^{*}=0.4, \alpha_{2}^{*}=0.4, \alpha_{3}^{*}=0.4, \alpha_{4}^{*}=0.7 \\
d_{1}^{-}=0, d_{1}^{+}=614.1110, d_{2}^{-}=1.2, d_{2}^{+}=0 \\
D^{*}=-612.9110 .
\end{gathered}
$$

Step 4: The main FFLP problem is found to be

$$
\begin{gathered}
\text { Min } D=d_{1}^{-}-d_{1}^{+}+d_{2}^{-}-d_{2}^{+} \\
\text {s.t. } \quad 25 x_{1}+27 x_{2}+30 x_{3}+28 x_{4}+d_{1}^{-}-d_{1}^{+}=5000 \\
\alpha_{1}+\alpha_{2}+\alpha_{3}+\alpha_{4}+d_{2}^{-}-d_{2}^{+}=4 \\
4.3 x_{1}+8 x_{2}+5.7 x_{3}+12 x_{4} \leq 1200+350\left(1-\alpha_{1}\right) \\
10 x_{1}+3.5 x_{2}+7 x_{3}+5 x_{4} \leq 2300+200\left(1-\alpha_{2}\right)(32) \\
13 x_{1}+15.2 x_{2}+4 x_{3}+9.1 x_{4} \leq 1700+400\left(1-\alpha_{3}\right) \\
11 x_{1}+7 x_{2}+14 x_{3}+10 x_{4} \leq 4000+800\left(1-\alpha_{4}\right) \\
10 \leq x_{1} \leq 100,3 \leq x_{2} \leq 100,12 \leq x_{3} \leq 100,5 \leq x_{4} \leq 100, \\
0.4 \leq \alpha_{1} \leq 1,0.4 \leq \alpha_{2} \leq 1,0.4 \leq \alpha_{3} \leq 1,0.4 \leq \alpha_{4} \leq 1, \\
x_{1} \geq 0, x_{2} \geq 0, x_{3} \geq 0, x_{4} \geq 0, d_{1}^{-} \geq 0, d_{1}^{+} \geq 0, d_{2}^{-} \geq 0, d_{2}^{+} \geq 0 .
\end{gathered}
$$

The obtained optimal solution

$$
x_{1}^{*}=76.77590, x_{2}^{*}=17, x_{3}^{*}=100, x_{4}^{*}=31.15530,
$$

and the optimal value of the objective function is $Z^{*}=6250.746$.

\section{Conclusion}


We considered a new version of fuzzy mathematical programming problem entitled Fuzzy Flexible Linear Programming (FFLP) problem to formulate a main practical problem. We saw the model to be more adaptive in practical situations. In particular, based on a pioneering concept of feasibility of solutions, a new concept of $\bar{\alpha}$-feasibility and $\bar{\alpha}$-efficiency of solution in fuzzy flexible linear programming problem was introduced to propose a parametric approach for solving the original problem by solving two associated classical linear programming problems. The usual available parametric approach needs to solve the main problem in two phases, not being convenient for the decision makers. We used sensitivity analysis for the suggested approach by considering for both phases of the mentioned approach. We proposed two methods entitled Algorithm1 and Algorithm 2. The results indicate that our proposed method (MPLGP) is more convenient and more applicable, since it can solve the problem directly in single process. To evaluate the efficiency of the proposed approach, we illustrated our discussion with a practical case based on post optimality for the parameters of the problem. We gave our examinations in five main categories. In the first case, we considered the amounts of $\alpha_{1}^{D}$ and $\alpha_{2}^{D}$ to be equal to 1 , while the third parameter $\alpha_{3}^{D}$ belonged to $[0.1,1]$. This illustrative examination showed that the corresponding MPLGP model to problem (25) provided various values of the objective function, decreasing from 118.5714 to 99.2851. Table 1 showed this result.

In the next examination, we considered the variation of the objective function value based on the variation of the parameter $\alpha_{2}^{D}$, which is allowed to change from 0.1 to 1 with a fixed value of $\alpha_{1}^{D}$ and $\alpha_{3}^{D}$ as $\alpha_{1}^{D}=\alpha_{3}^{D}=1$. Various values of the objective function for the MPLGP model associated with problem (25) were obtained as reported in Table 2. According to this table, we see that there is not any improvement on the objective function value, based on variation of the parameter $\alpha_{2}^{D}$. In fact, we conclude that increase in the resource level corresponding to the second constraint is not effective in improving the optimal value of the objective function.

In the third examination, we explored the variation of the objective function value based on the variation of the first lower bound of the satisfaction level, that is, $\alpha_{1}^{D}$, with fixed values for the second and the third levels as $\alpha_{2}^{D}=\alpha_{3}^{D}=1$. The achieved numerical results from solving the MPLGP model associated with problem (25) was given in Table 3.

We see that unlike the previous examined case, in the current case, any increase in the resource level corresponding to the first constraint is strongly effective beyond the degree of satisfaction equal to 0.5 . Hence, any decrease in the degree of satisfaction of the first constraint from 0.5 to zero will result on a great improvement on the optimal value of the objective function. In particular, the above numerical examination showed that variation on the resource level for the second constraint is not effective to improve the current optimal value of the objective function, while the objective function value for sensitive upon variation on the available resources for the first and the third constraints. This lead us to focus on a new examination for the decision maker to vary the optimal value of the objective function for any variation of the first and the third available resource levels simultaneously. Table 4 gives the result of this examination. Clearly, Table 4 gave the various values of the objective function of the MPLGP for problem (25) based on the variation from 0.1 to 1 for $\alpha_{1}^{D}$ and $\alpha_{3}^{D}$. The results in Table 4 showed that the optimal value of the objective function with 
respect to the parameter $\alpha_{3}^{D}$ was more sensitive in comparison with the parameter $\alpha_{1}^{D}$. As a final examination of our numerical study, we made a discussion on sensitivity analysis when a decision maker would like to improve the satisfaction level for variation of all parameters. We assume that the decision maker would like to make an optimal decision for the MPLGP problem associated with problem (25) while the summation value of the satisfaction level of all the constraints were to be equal or bigger than some desired values. The obtained results showed that the proposed model could obtain an optimal solution with the same value for the objective function of problem (25) with a higher degree for the summation of the parameters $\alpha_{i}, i=1,2,3$. According to the obtained optimal solution for the resulting model, we obtained a better amount for the objective function of the first problem as compared to the two-phase model. Also, a better value of $\sum_{i=1}^{3} \alpha_{i}$, was obtained. Finally, the above illustrative examples and in particular, the given case study showed our proposed approach to be more convenient for the decision maker, because it could solve the fuzzy flexible linear programming problem using more convenient steps. Furthermore, the same approach may be applied when to an extended model with fuzzy tolerances and to the generalized model with as multi-objectives having fuzzy flexible constraints.

\section{Acknowledgment.}

We would like to appreciate the constructive comments of the anonymous referees and the valuable suggestions of Editor-in-Chief of IJOR, Prof. Nezam Mahdavi-Amiri have who been helped us for improving the earlier versions of the paper.

\section{References}

[1] Attari, H. and S.H. Nasseri, S.N. (2014), New concepts of feasibility and efficiency of solutions in fuzzy mathematical programming problems, Fuzzy Information and Engineering, 6, 203-221.

[2] Bector, C. R, Chandra, S. (2005), Fuzzy mathematical programming and fuzzy matrix games. In: Studies in Fuzziness and Soft Computing, vol.169. Springer, Berlin

[3] Bellman, R.E. and Zadeh, L.A. (1970), Decision making in a fuzzy environment, Management Science, 17, 141-182.

[4] Cal, T. (1995), Post Optimal Analysis, Parametric Programming and Related Topics, De gruyter, New York.

[5] Chang, C.-T. (2004), On the mixed binary goal programming problems. Applied Mathematics and Computation 159, 759-768.

[6] Chang, C.-T. (2007), Multi-choice goal programming. Omega, 35, 389-396.

[7] Chang, C.-T. (2008), Revised multi-choice goal programming. Applied Mathematical Modelling, 32, 2587-2595.

[8] Charnes, A. and Cooper, W.W. (1959), Constraint programming, Management Science, 6, 73-79. 
[9] Compose, L. and Verdegay, J.L, J.L. (1989), Linear programming problems and ranking of fuzzy number, Fuzzy Sets and Systems, 32, 1-11.

[10] Delgado, M., Herrera, F. Verdegay, J.L. and Vila, M.A.(1993), Post-optimality analysis on the membership function of a fuzzy linear programming problem, Fuzzy Sets and Systems, 53, 289-297.

[11] Delgado, M., Verdegay, J.L. and M.A. Vila, M.A. (1989), A general model for fuzzy linear programming, Fuzzy Sets and Systems, 29, 21-29.

[12] Dubois, D. and Prade, H. (1980), Fuzzy Sets and Systems, Theory and Application, Academic press, New York.

[13] Dubois, D. and Prade, H. (1980), Ranking fuzzy members in the setting of possibility theory, Information Science, 30, 183-224.

[14] Dubois, D. and Prade, H. (1982), System of linear fuzzy constraints, Fuzzy Sets and Systems, 13, 43-52.

[15] Fangs, S.C. and C.F. Hu, C.F. (1999), Linear programming with fuzzy coefficients in constraint, Compute. Math. Appl, 37, 63-76.

[16] Fiacco, A.V. (1989), Introduction to sensitivity and stability analysis in nonlinear programming, Academic Press, New York.

[17] Fortemps, P. and Rubnes, M. (1996), Ranking and defuzzification methods based on area compensation, Fuzzy Sets and Systems, 82, 319-330.

[18] Ignizio, J.P. (1984), Linear programming in single-and multiple -objective systems, Journal of Policy Analysis and Management 3(3), 477-489.

[19] Lee, S.M. (1972), Goal programming for Decision Analysis, Auerback, Philadelphia.

[20] Mahdavi-Amiri, N. and Nasseri, S.H. (2007), Duality results and a dual simplex method for linear programming problem with trapezoidal fuzzy variables, Fuzzy Sets and Systems, 158, 1961-1978.

[21] Maleki, H.R., Tata, M. and Mashinchi, M. (2000), Linear programming with fuzzy variables, Fuzzy Sets and Systems, 109, 21-33.

[22] Narasimhan, R. (1980), Goal programming in a fuzzy environment, Decision Science $1980 ; 11,243-252$.

[23] Nasseri, S.H. and Ebrahimnejad, A. (2010), A fuzzy optimal simplex algorithm and its application for solving flexible linear programming problems, European Journal of Industrial Engineering, 4(3), 372-389.

[24] Nasseri, S.H. and E. Behmanesh, E. (2013), Linear programming with triangular fuzzy numbers: a case study in a finance and credit in state, Fuzzy Information and Engineering, $5,253-272$. 
[25] Nasseri, S.H., Attari, H. and Ebrahimnejad, A. (2012), revised simplex method and its application for solving fuzzy linear programming problems, European Journal of Industrial Engineering, 6(3), 259-280.

[26] Nasseri, S.H., Behmanesh, E., Taleshian, F., Abdolalipour, M. and. Taghi-Nejad, N. (2013), Fully fuzzy linear programming with inequality constraint, International Journal of Industrial Mathematics, 5(4), 309-316.

[27] Romero. (2001), Extended Lexicographic goal programming: unifying approach, Omega, 29, 63-71.

[28] Rommelfanger, H., Hanuscheck, R. and Wolf, J. (1989), Linear programming with fuzzy objective, Fuzzy Sets and Systems, 29, 31-48.

[29] Rubnes, M. (1991), Inequality constraints between fuzzy numbers and their use in mathematical programming, in: R. Slowinski, J. Teghem (Eds.), Stochastic versus fuzzy approaches to multi-objective mathematical programming under uncertainty, Kluwer Academic Publishers, Dordrecht, 321-330.

[30] Sakawa, N. and H. Yana, H. (1985), Interactive decision making for multi-objective linear fractional programming problems with fuzzy parameters, Cybernetics System, 16, 377-397.

[31] Tabrizi, B.B., Shahanaghi, K. and Jabalameli, M.S. (2012), Fuzzy multi-choice goal programming. Applied Mathematical Modelling, 36: 1415-1420.

[32] Tanaka, H. and Assai, K. (1984), Fuzzy linear programming problems with fuzzy number, Fuzzy Sets and Systems, 13, 1-10

[33] Verdegay, J.L. (1982), Fuzzy mathematical programming, in M.M. Gupata and E. sanches, Eds, Fuzzy Information and Decision Processes, (North-Holland, Amsterdam) 231-237.

[34] Verdegay, J.L. (1984), a dual approach to solving the fuzzy linear programming problem, Fuzzy Sets and Systems, 14, 131-141.

[35] Wang. X. and E. Kerre, E. (2001), Reasonable properties for the ordering of fuzzy quantities (2 parts), Fuzzy Sets and Systems, 118, 375-405.

[36] Werner's, B. (1987), Interactive multiple objective programming subject to flexible constraint, European Journal of Operation Research, 31, 342-349.

[37] Yager, R.R. (1981), A procedure for ordering fuzzy subsets of the unit interval, Information Science, 24, 143-161.

[38] Zadeh, L.A. (1987), Fuzzy sets as a basis for a theory of possibility, Fuzzy Sets and Systems, 1, 3-28.

[39] Zimmermann, H.J. (1978), Fuzzy programming and linear programming with several objective function and, Fuzzy Sets and Systems, 1, 45-55. 
